\title{
Accelerating infall and rotational spin-up in the hot molecular core G31.41+0.31
}

\author{
M. T. Beltrán ${ }^{1}$, R. Cesaroni ${ }^{1}$, V. M. Rivilla ${ }^{1}$, Á. Sánchez-Monge ${ }^{2}$, L. Moscadelli ${ }^{1}$, A. Ahmadi ${ }^{3}$, V. Allen ${ }^{4,5}$, \\ H. Beuther ${ }^{3}$, S. Etoka ${ }^{6}$, D. Galli ${ }^{1}$, R. Galván-Madrid ${ }^{7}$, C. Goddi ${ }^{8,9}$, K. G. Johnston ${ }^{10}$, P. D. Klaassen ${ }^{11}$, \\ A. Kölligan ${ }^{12}$, R. Kuiper ${ }^{12}$, M. S. N. Kumar ${ }^{13,14}$, L. T. Maud ${ }^{15,9}$, J. C. Mottram ${ }^{3}$, T. Peters ${ }^{16}$, P. Schilke ${ }^{2}$, L. Testi ${ }^{1,17}$, \\ F. van der Tak ${ }^{4,5}$, and C. M. Walmsley ${ }^{\star}$
}

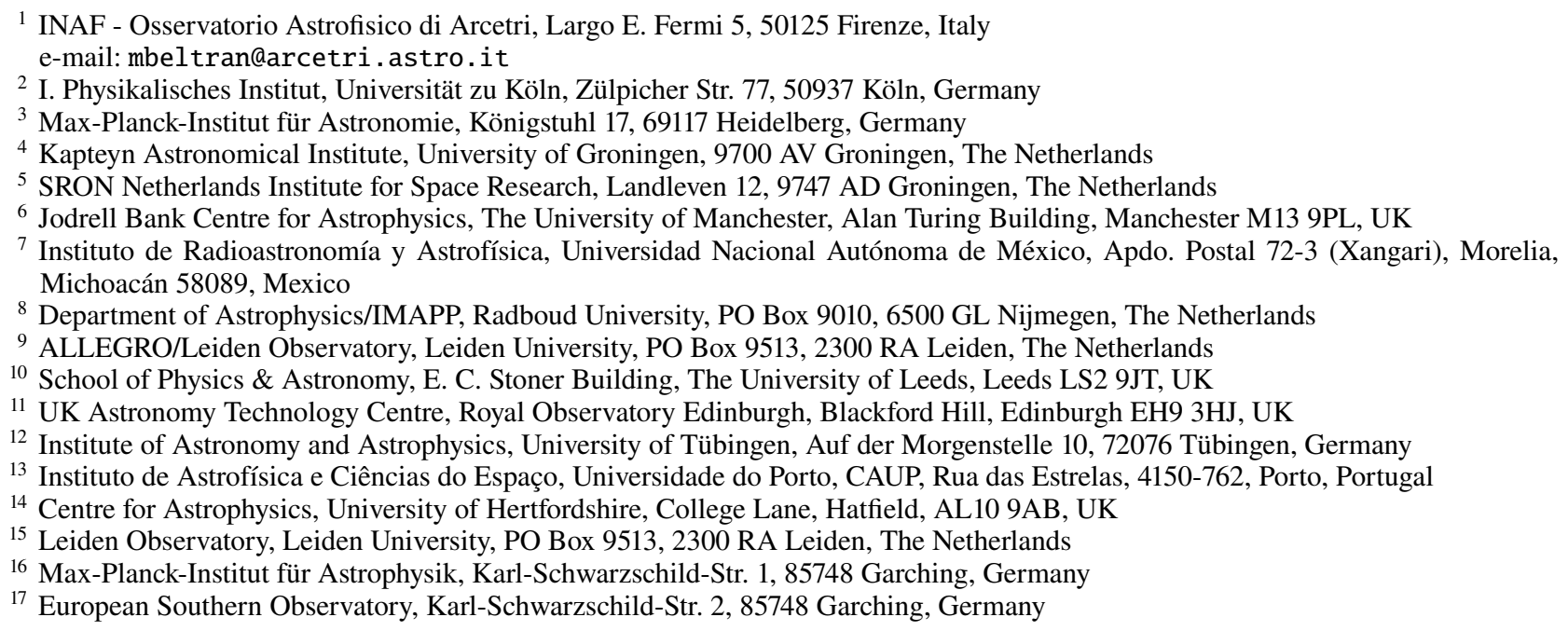

Received 12 February 2018 / Accepted 14 March 2018

\begin{abstract}
As part of our effort to search for circumstellar disks around high-mass stellar objects, we observed the well-known core G31.41+0.31 with ALMA at $1.4 \mathrm{~mm}$ with an angular resolution of $\sim 0$ '.22 ( 1700 au). The dust continuum emission has been resolved into two cores namely Main and NE. The Main core, which has the stronger emission and is the more chemically rich, has a diameter of $\sim 5300$ au, and is associated with two free-free continuum sources. The Main core looks featureless and homogeneous in dust continuum emission and does not present any hint of fragmentation. Each transition of $\mathrm{CH}_{3} \mathrm{CN}$ and $\mathrm{CH}_{3} \mathrm{OCHO}$, both ground and vibrationally excited, as well as those of $\mathrm{CH}_{3} \mathrm{CN}$ isotopologues, shows a clear velocity gradient along the NE-SW direction, with velocity linearly increasing with distance from the center, consistent with solid-body rotation. However, when comparing the velocity field of transitions with different upper level energies, the rotation velocity increases with increasing energy of the transition, which suggests that the rotation speeds up toward the center. Spectral lines towardtoward the dust continuum peak show an inverse P-Cygni profile that supports the existence of infall in the core. The infall velocity increases with the energy of the transition suggesting that the infall is accelerating toward the center of the core, consistent with gravitational collapse. Despite the monolithic appearance of the Main core, the presence of red-shifted absorption, the existence of two embedded free-free sources at the center, and the rotational spin-up are consistent with an unstable core undergoing fragmentation with infall and differential rotation due to conservation of angular momentum. Therefore, the most likely explanation for the monolithic morphology is that the large opacity of the dust emission prevents the detection of any inhomogeneity in the core.
\end{abstract}

Key words. ISM: individual objects: G31.41+0.31 - ISM: jets and outflows - ISM: molecules - stars: formation techniques: interferometric

\section{Introduction}

The formation process of high-mass stars has puzzled the astrophysical community for decades because of the apparent stellar

\footnotetext{
${ }^{\star}$ Malcolm Walmsley could not see the completion of this article, as he passed away a few months ago. However, his contribution to our project was crucial and will never be forgotten.
}

mass limit for spherical accretion. Beyond this limit, theory predicted that it is impossible to continue accreting material because the stellar wind and the radiation pressure from the newly-formed early-type star would stop the infall (e.g., Kahn 1974; Yorke \& Krügel 1977; Wolfire et al. 1987). Different theoretical scenarios have proposed nonspherical accretion as a possible solution for the formation of OB-type stars (Nakano et al. 1989; Jijina \& Adams 1996), and in recent years, all models 
appear to have converged to a disk-mediated accretion scenario (e.g., Krumholz et al. 2009; Kuiper et al. 2010, 2011; Peters et al. 2010a; Kuiper \& Yorke 2013; Klassen et al. 2016). Competing theories that propose very different high-mass star-formation mechanisms, such as models suggesting that massive starformation is initiated by the monolithic collapse of a turbulent molecular core (McKee et al. 2002), or those based on competitive accretion (Bonnell\& Bate 2006), all predict the existence of circumstellar accretion disks through which the material is channeled onto the forming star. High-angular resolution observations have confirmed these theoretical prediction and, in recent years, especially since the advent of ALMA, circumstellar disks around B-type stars, which have luminosities $<10^{5} L_{\odot}$, have been discovered. In some cases, it has been proposed that these structures could be true accretion disks undergoing Keplerian rotation (see the review by Beltrán \& de Wit 2016). The detection of Keplerian disk candidates has been recently extended up to more massive late O-type stars, with luminosities of $\sim 10^{5} \mathrm{~L}_{\odot}$ (Johnston et al. 2015; Ilee et al. 2016).

From an observational point of view, the natural followup to these findings is to search for disks around early O-type stars, with spectral types earlier than O6-O7, and luminosities $>10^{5} L_{\odot}$, to test if stars of all masses could form through diskmediated accretion. With this in mind, we carried out a survey with ALMA of six O-type star-forming regions associated with known hot molecular cores (HMCs) looking for circumstellar disks. The first analysis of these observations has revealed different degrees of fragmentation of the cores and the presence of three Keplerian disk candidates (Cesaroni et al. 2017). One of the HMCs of this survey is G31.41+0.31 (hereafter G31), for which our ALMA observations have revealed no signs of fragmentation and a velocity gradient consistent with rotation.

The well-known HMC G31 has a luminosity $\gtrsim 10^{5} L_{\odot}$ (Cesaroni et al. 1994) and is located at a kinematic distance of $7.9 \mathrm{kpc}$ (Churchwell et al. 1990). Alternatively, G31 might be associated with the W43-Main cloud complex, as suggested by Nguyen-Luong et al. (2011). For W43-Main, two distance estimates exist which are based on VLBI observations of maser parallax. Reid et al. (2014) report a distance of $4.9 \mathrm{kpc}$ to the W43-Main core, Zhang et al. (2014) report distances to 5 maser spots ranging from 4.27 to $6.21 \mathrm{kpc}$. Given the uncertain association of G31 with W43-Main and the uncertainty in the distance to W43-Main itself, we adopt the kinematic distance of $7.9 \mathrm{kpc}$ for G31 in this paper. However, one should keep in mind that the real distance may be substantially less $(\sim 5 \mathrm{kpc})$ which would imply a $\sim 2.5$ times lower luminosity.

The G31 hot core has a size of $\sim 1^{\prime \prime}(\sim 8000 \mathrm{au})$ and its mass is $\gtrsim 500 M_{\odot}$ (Beltrán et al. 2004; Girart et al. 2009; Cesaroni et al. 2011). The core is $\sim 5^{\prime \prime}$ away from an ultracompact (UC) HII region and overlaps in projection a diffuse halo of free-free emission, possibly associated with the UC HII region itself. Given the intensity of the molecular line and continuum emission, the core has been extensively studied with both single-dish and interferometric observations (e.g., Cesaroni et al. 1994, 2011; Olmi et al. 1996; Beltrán et al. 2004, 2005; Girart et al. 2009; Mayen-Gijon et al. 2014). These observations, mostly at millimeter wavelengths, have confirmed that the core is very chemically rich, presenting prominent emission in a large number of complex organic molecules, some of them of prebiotic importance (e.g., Beltrán et al. 2009; Rivilla et al. 2017). The molecular emission has been successfully used to trace the gas distribution and kinematics of the dense, hot gas and estimate important physical parameters (e.g., temperature and density: Beltrán et al. 2005). Molecular

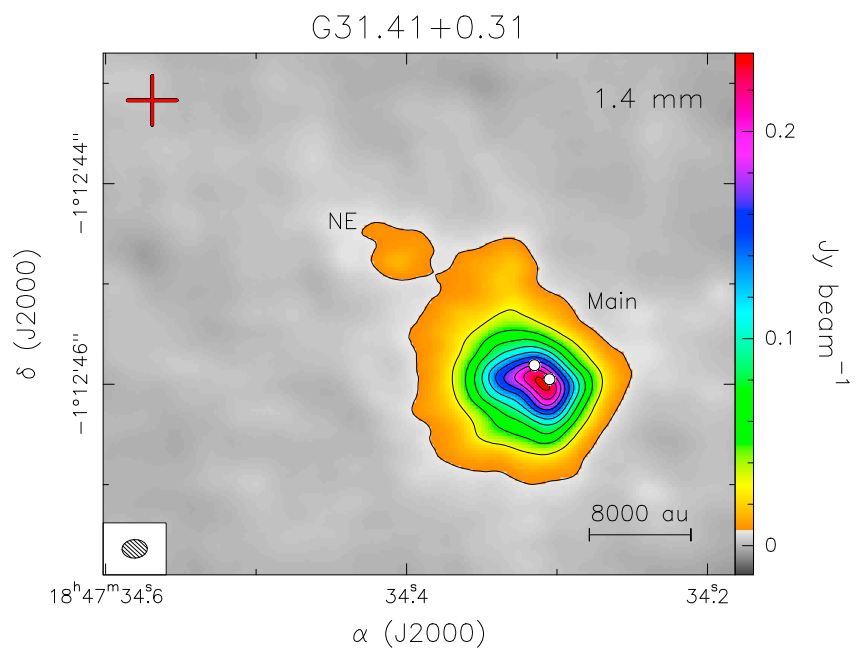

Fig. 1. ALMA map of the $1.4 \mathrm{~mm}$ continuum emission from the G31 HMC. The contours range from $7.5(5 \sigma)$ to $232.5 \mathrm{mJy} \mathrm{beam}^{-1}$ in steps of $22.5 \mathrm{mJy}$ beam $^{-1}(15 \sigma)$. The white dots mark the position of two unresolved free-free continuum sources detected at wavelengths between $3.6 \mathrm{~cm}$ and $7 \mathrm{~mm}$ by Cesaroni et al. (2010). The red cross indicates the position of the UC HII region. The synthesized beam is shown in the lower left-hand corner.

line observations have also revealed the existence of a striking velocity gradient (centered at an LSR velocity of $\sim 96.5 \mathrm{~km} \mathrm{~s}^{-1}$ ) across the core in the northeast-southwest (NE-SW) direction (Beltrán et al. 2005; Cesaroni et al. 2011). This has been interpreted as rotational motion around embedded massive stars, as suggested by the detection of two point-like $(<0.07)$ freefree continuum sources close to the core center (Cesaroni et al. 2010). Polarization measurements by Girart et al. (2009) have revealed an hour-glass shaped magnetic field of $\sim 10 \mathrm{mG}$, with the symmetry axis oriented perpendicular to the velocity gradient. Moreover, the same authors have detected inverse P-Cygni profiles in a few molecular lines, indicating that infall motion is present (see also Mayen-Gijon et al. 2014). Wyrowski et al. (2012) have also detected red-shifted absorption in ammonia with SOFIA. All these features are consistent with a scenario in which the core is contracting and rotating about the direction of the magnetic field lines.

In this work, we analyze in more detail the ALMA observations of G31 previously presented by Cesaroni et al. (2017). These observations have an angular resolution of $\sim 0 . ' 22$ ( $\sim 1700$ au at the distance of the source), which is about four times higher than that of previous (sub)millimeter observations (e.g., Beltrán et al. 2005; Girart et al. 2009; Cesaroni et al. 2011) and therefore allow us to study the morphology and kinematics of this core with unprecedented detail. In particular, we investigate whether the compact appearance of G31, with no hint of fragmentation despite its large mass, is real. We study the physical conditions of the core and of the molecular outflows associated with it. Regarding the kinematics, by analyzing the velocity gradient, we discuss what type of rotation the core could be undergoing, and by analyzing the red-shifted absorption detected toward the center of the core, we discuss its possible collapse.

\section{Observations}

Interferometric observations of G31 were carried out with ALMA in Cycle 2 in July and September 2015 as part of project 2013.1.00489.S. (P.I.: R. Cesaroni). The observations 
Table 1. Positions, flux densities, and diameters of the cores.

\begin{tabular}{|c|c|c|c|c|c|c|c|c|}
\hline \multirow[b]{2}{*}{ Core } & \multicolumn{2}{|c|}{ Peak position } & \multirow[b]{2}{*}{$\begin{array}{l}T_{\mathrm{B}}^{a} \\
(\mathrm{~K}) \\
\end{array}$} & \multirow[b]{2}{*}{$\begin{array}{c}I_{v}^{\text {peak }, b} \\
\left(\mathrm{Jy} \mathrm{beam}^{-1}\right)\end{array}$} & \multirow[b]{2}{*}{$\begin{array}{c}S_{v}^{b} \\
(\mathrm{Jy})\end{array}$} & \multicolumn{3}{|c|}{ Source diameter } \\
\hline & $\begin{array}{c}\alpha(\mathrm{J} 2000) \\
\text { h m s }\end{array}$ & $\begin{array}{c}\delta(\mathrm{J} 2000) \\
\circ, \prime \prime\end{array}$ & & & & $\begin{array}{l}\mathrm{FWHM}^{c} \\
(\operatorname{arcsec})\end{array}$ & $\begin{array}{c}\theta_{s}^{d} \\
(\operatorname{arcsec})\end{array}$ & $\begin{array}{c}D_{s}^{d} \\
(\mathrm{au})\end{array}$ \\
\hline G31-Main & 184734.309 & -011245.99 & $132 \pm 1$ & $0.238 \pm 0.002$ & $3.10 \pm 0.05$ & $0.70 \pm 0.04$ & $0.67 \pm 0.04$ & $5300 \pm 300$ \\
\hline G31-NE & 184734.407 & -011244.79 & $8 \pm 1$ & $0.015 \pm 0.002$ & $0.052 \pm 0.002$ & $0.61 \pm 0.04$ & $0.56 \pm 0.04$ & $4500 \pm 300$ \\
\hline
\end{tabular}

Notes. Derived from the continuum dust emission. ${ }^{(a)}$ The conversion factor from Jy beam ${ }^{-1}$ to $\mathrm{K}$ is 555.5. ${ }^{(b)}$ Peak intensity and integrated flux density corrected for primary beam response. ${ }^{(c)} F W H M=2 \sqrt{A / \pi}$, where $A$ is the area inside the contour at half maximum. ${ }^{(d)}$ Deconvolved diameter, calculated assuming that the cores are symmetric Gaussians, using the expression $\theta_{s}=\sqrt{F W H M^{2}-H P B W^{2}}$, where $H P B W$ is the half-power width of the synthesized beam. The distance to G31 is assumed to be $7.9 \mathrm{kpc}$.

were performed in Band 6 with the array in an extended configuration. The digital correlator was configured in thirteen spectral windows (SPW) that cover among other lines, $\mathrm{SiO}(5-4)$, $\mathrm{CH}_{3} \mathrm{CN}(12-11), \mathrm{CH}_{3}{ }^{13} \mathrm{CN}(12-11), \mathrm{CH}_{3} \mathrm{CN} v_{8}=1$ (12-11), and ${ }^{13} \mathrm{CH}_{3} \mathrm{CN}$ (13-12). We refer to Cesaroni et al. (2017) for detailed information on the observations, and, in particular, on the continuum subtraction.

The phase reference center of the observations is $\alpha(\mathrm{J} 2000)$ $=18^{\mathrm{h}} 47^{\mathrm{m}} 34.315, \delta(\mathrm{J} 2000)=-01^{\circ} 12^{\prime} 45^{\prime \prime} \cdot 90$. The position uncertainty is $<0$ ' 2 . The data were calibrated and imaged using the $\mathrm{CASA}^{1}$ software package (McMullin et al. 2007). Further imaging and analysis were done with the GILDAS ${ }^{2}$ software package. A total of 13 individual data cubes were created using the CLEAN task and the ROBUST parameter of Briggs (1995) set equal to 0.5 . The continuum was determined from the broadest spectral window, centered at $218 \mathrm{GHz}$, using the STATCONT algorithm (Sánchez-Monge et al. 2018). The resulting synthesized CLEANed beam of the maps is $0{ }^{\prime} .25 \times 00^{\prime} .19$ for the continuum, and ranges from $0 .{ }^{\prime} 25 \times 0.0^{\prime} 19$ to $0.27 \times 0 . ' 20$ for the lines analyzed in this work. These angular resolutions correspond to spatial scales of $\sim 1700 \mathrm{au}$ at the distance of the source. The rms noise of the maps is $1.5 \mathrm{mJy}^{-1}$ beam $^{-1}$ for the continuum and $\sim 1.3 \mathrm{mJy}$ beam $^{-1}$ per channel of $2.7 \mathrm{~km} \mathrm{~s}^{-1}, \sim 1.6 \mathrm{mJy} \mathrm{beam}^{-1}$ per channel of $0.66 \mathrm{~km} \mathrm{~s}^{-1}$, and $\sim 2.1 \mathrm{mJy}^{-1}$ beam $^{-1}$ per channel of $0.33 \mathrm{~km} \mathrm{~s}^{-1}$ for the line maps. The total bandwidth of the spectral window used to estimate the continuum is $1875 \mathrm{MHz}$ and the spectral resolution is $\sim 1.95 \mathrm{MHz}$. The number of channels with emission considered to be continuum by STATCONT ranges from 144, which corresponds to a bandwidth of $280 \mathrm{MHz}$, at line-poor positions, to 10 , which corresponds to a bandwidth of $17 \mathrm{MHz}$, at line-rich positions. However, the rms noise of the continuum map would indicate an effective bandwidth of $\sim 2 \mathrm{MHz}$ if the noise were just thermal noise. This along with the fact that the continuum source is very strong leads us to conclude that the high rms noise of such map is due to dynamic range problems and not to the STATCONT method used to subtract the continuum.

\section{Results}

\subsection{Continuum emission}

Figure 1 shows the continuum emission map of the G31 hot core at $1.4 \mathrm{~mm}(217 \mathrm{GHz})$. The dust emission is resolved into two cores, a strong and large one located at the center of the map that

\footnotetext{
1 The CASA package is available at http://casa.nrao.edu/

2 The GILDAS package is available at http://www.iram.fr/ IRAMFR/GILDAS

3 http://www . astro. uni-koeln. de/ sanchez/statcont
}

we call Main, and a smaller and weaker one located to the northeast of the Main core, which we call NE. The UC HII region, located to the northeast of the HMC, is not detected in the continuum emission at $1.4 \mathrm{~mm}$. The position, flux, and diameter of the cores are given in Table 1. The angular diameter has been computed as the diameter of the circle whose area equals that inside the $50 \%$ contour level.

The dust continuum emission of the Main core peaks close to the position of one of the two unresolved free-free continuum sources detected from $3.6 \mathrm{~cm}$ to $7 \mathrm{~mm}$ by Cesaroni et al. (2010). As seen in Fig. 1, this core appears quite round, uniform, and compact, with no hint of fragmentation, despite the fact that the angular resolution of the observations is high enough to properly resolve the emission. In fact, the deconvolved half-power diameter of the core is $\sim 5300 \mathrm{au}$, much larger than the spatial resolution of the observations $(\sim 1700 \mathrm{au})$. The deconvolved diameter of the source at the $5 \sigma$ emission level is $\sim 17000 \mathrm{au}$, ten times larger than the synthesized beam. By fitting elliptical Gaussians to the dust emission map, we measured a deconvolved size of the source of $0 . ' 9 \times 0 . .7(7000 \times 5500 \mathrm{au})$ with a position angle (PA) of $\sim 63^{\circ}$.

The NE core is much weaker than the Main core. Its integrated flux density is $\sim 60$ times lower and its peak intensity $\sim 16$ times lower. This source is much smaller than the Main core and has a $5 \sigma$ emission level deconvolved diameter of $\sim 4500 \mathrm{au}$, which coincides with the deconvolved diameter of the $50 \%$ contour level.

\subsection{Line emission}

Figures 2 and A.1 show the spectra observed in the 13 spectral windows toward the Main and the NE core, respectively. The spectra were obtained by integrating over the $5 \sigma$ contour level area of the dust continuum emission. Clearly, the Main core is the most chemically rich, since the corresponding spectrum displays a forest of molecular lines. For this reason, we postpone a detailed analysis of the molecular line emission toward this core to a future study (Rivilla et al., in prep.). In the present work, we focus on i) two well-known hot core (high-density) tracers: $\mathrm{CH}_{3} \mathrm{CN}$ (ground state and vibrationally excited) and its isotopologues $\mathrm{CH}_{3}{ }^{13} \mathrm{CN}$ and ${ }^{13} \mathrm{CH}_{3} \mathrm{CN}$, and $\mathrm{CH}_{3} \mathrm{OCHO}$ (ground state and vibrationally excited), and ii) a typical outflow tracer: $\mathrm{SiO}$. Occasionally, we also discuss the emission of the lower density tracer $\mathrm{H}_{2} \mathrm{CO}$. The different transitions of these species are indicated in Figs. 2 and A.1. The typical cloud and/or molecular outflow tracers $\mathrm{C}^{18} \mathrm{O}$ and ${ }^{13} \mathrm{CO}$ are also covered by our spectral setup but their emission is so heavily filtered out by the interferometer that it has been impossible to use these lines for our study. 
G31.41+0.31 - Main
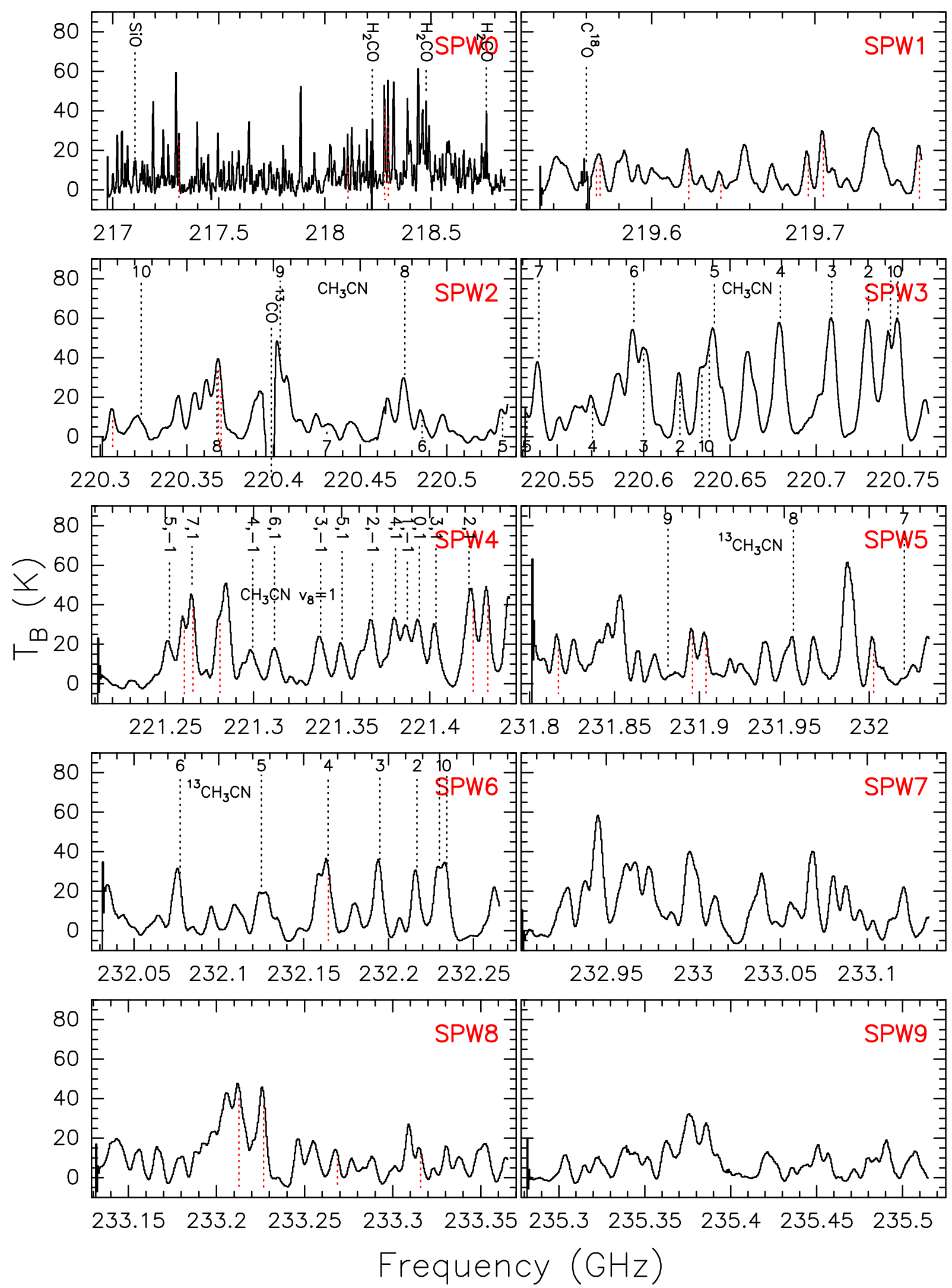

Fig. 2. Continuum-subtracted spectra obtained by integrating the emission over the $5 \sigma$ contour level area of the continuum emission of the Main core in G31. The spectra shown are spread over the whole frequency range of the ALMA observations. Different $K$ numbers are marked with dotted lines in the upper part of each spectra in the case of $\mathrm{CH}_{3} \mathrm{CN}$ (SPW2 and 3), vibrationally excited $\mathrm{CH}_{3} \mathrm{CN}$ (SPW4), and ${ }^{13} \mathrm{CH}_{3} \mathrm{CN}$ (SPW5 and 6), and in the lower part in the case of $\mathrm{CH}_{3}{ }^{13} \mathrm{CN}$ (SPW2 and 3). The red dotted lines in the lower part of each spectra indicate $\mathrm{CH}_{3} \mathrm{OCHO} v=0$ and $v_{t}=1$ transitions. The different spectral windows (SPW) are indicated in red in the top right of each panel. 

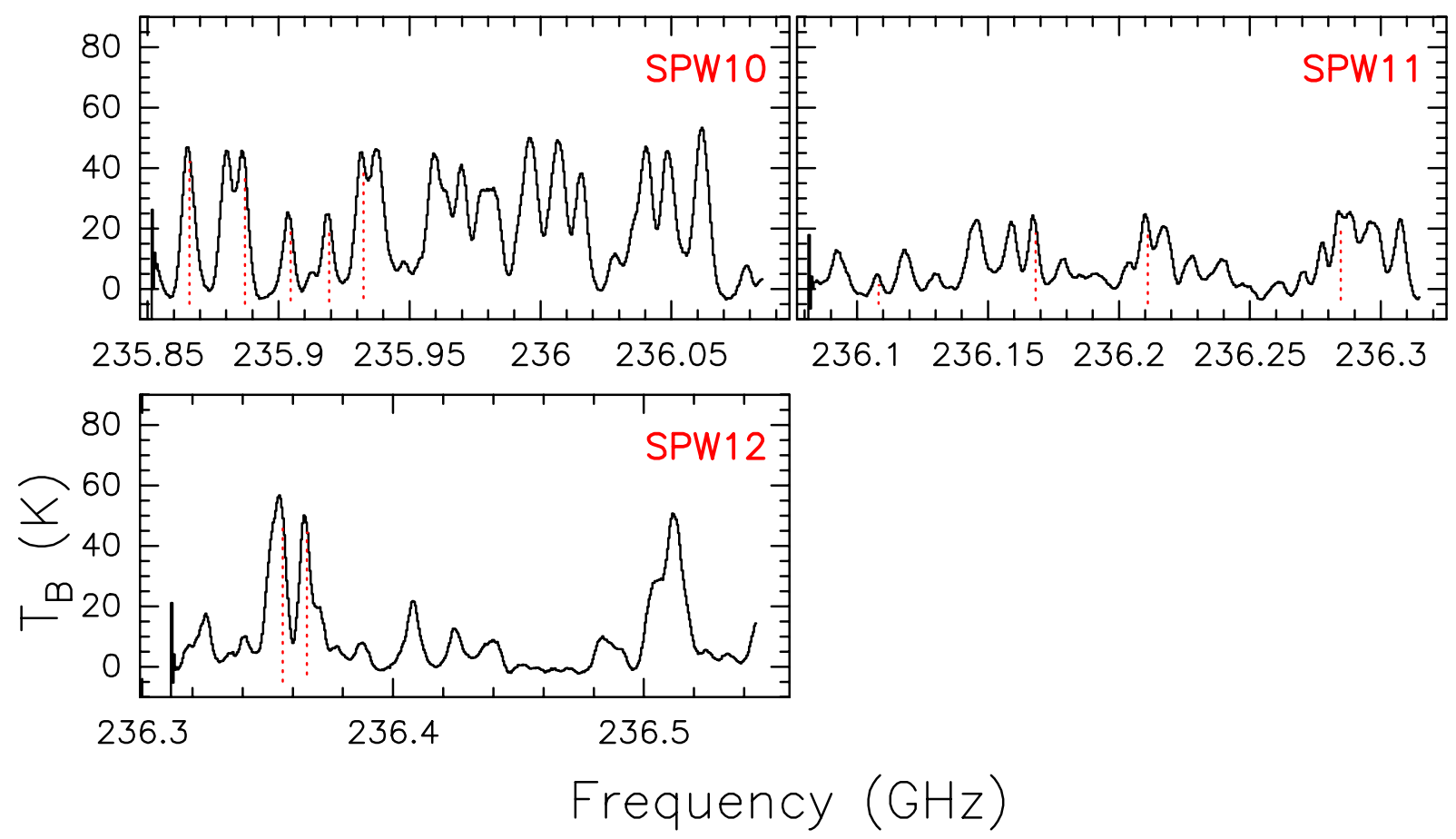

Fig. 2. continued
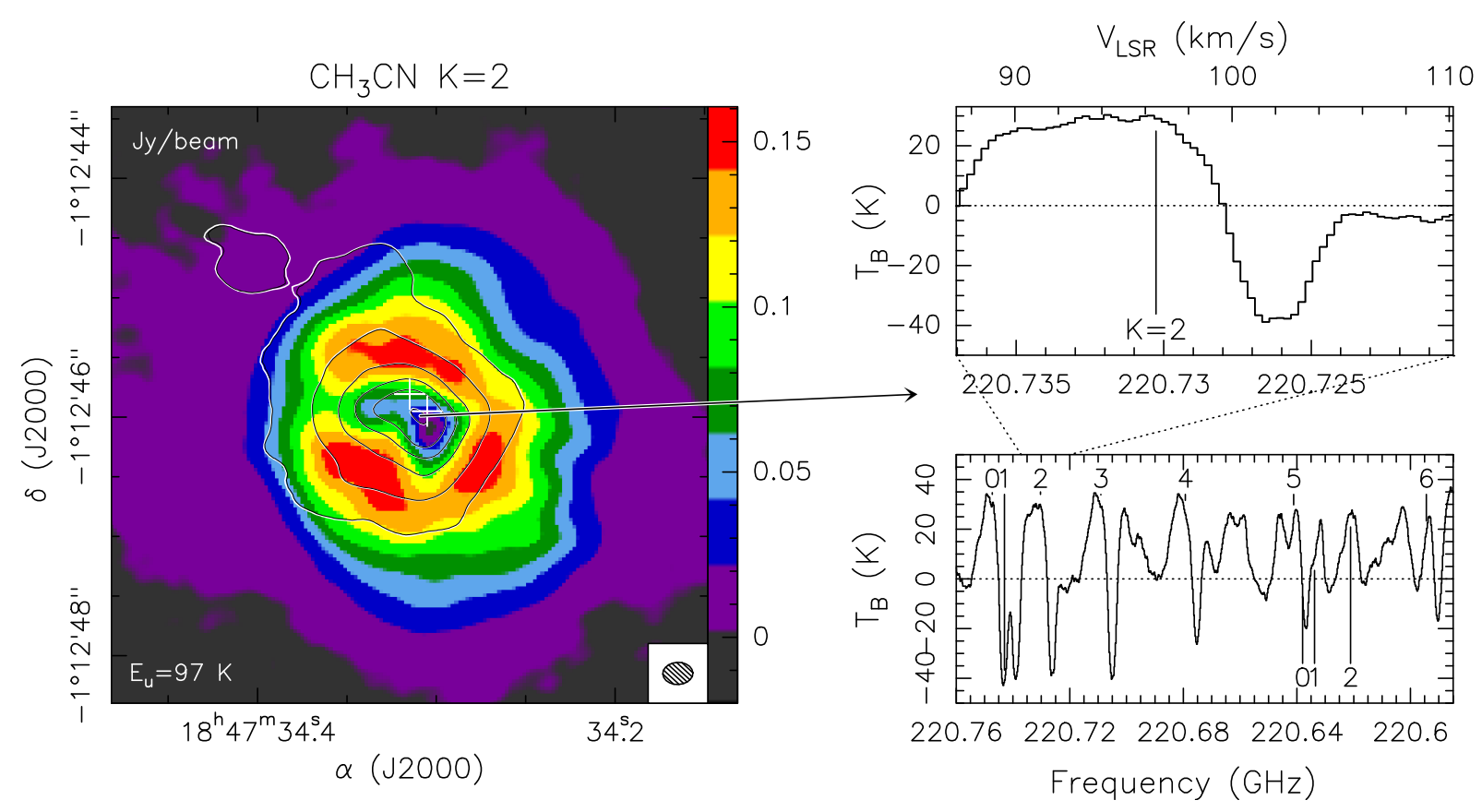

Fig. 3. Left panel: dust continuum emission map (contours) overlaid on the map of the $\mathrm{CH}_{3} \mathrm{CN}(12-11) K=2$ emission averaged over the velocity range 92 to $102 \mathrm{~km} \mathrm{~s}^{-1}$ (colors). Contours are 7.5, 30, 75, 120, 165, and $232.5 \mathrm{mJy} \mathrm{beam}^{-1}$. The white crosses indicate the positions of the two compact free-free continuum sources detected by Cesaroni et al. (2010). The synthesized beam is shown in the lower right-hand corner. Right panels: spectra of the $\mathrm{CH}_{3} \mathrm{CN} K=0$ to 6 and $\mathrm{CH}_{3}{ }^{13} \mathrm{CN} K=0$ to 2 toward the absorption dip, that is, toward the dust continuum peak. The upper right panel zooms in on the $\mathrm{CH}_{3} \mathrm{CN} K=2$ spectrum.

\subsection{1. $\mathrm{CH}_{3} \mathrm{CN}$ and isotopologues, $\mathrm{CH}_{3} \mathrm{OCHO}$, and $\mathrm{H}_{2} \mathrm{CO}$}

The different $K$ transitions of $\mathrm{CH}_{3} \mathrm{CN}$ (including the vibrationally excited transitions) and the two isotopologues $\mathrm{CH}_{3}{ }^{13} \mathrm{CN}$, and ${ }^{13} \mathrm{CH}_{3} \mathrm{CN}$ cover a broad range of excitation conditions, with upper level energies ranging from $\sim 70 \mathrm{~K}$ to $\sim 860 \mathrm{~K}$. On the other hand, our setup covers almost 2000 transitions of $\mathrm{CH}_{3} \mathrm{OCHO}$, including both ground and vibrationally excited, with upper level energies of $\sim 20-1700 \mathrm{~K}$. In our data, many of these transitions are strongly blended with other species (and some are simply too faint to be detected). In the end we have analyzed only those transitions of $\mathrm{CH}_{3} \mathrm{OCHO}$ that are unblended or slightly blended with other species, which in total number are $\sim 40$ transitions. These transitions cover a range of energies of $\sim 100-440 \mathrm{~K}$.

These high-density tracers have allowed us to study the emission of the hot core at different excitation conditions, likely tracing material at different depths in the core because one 


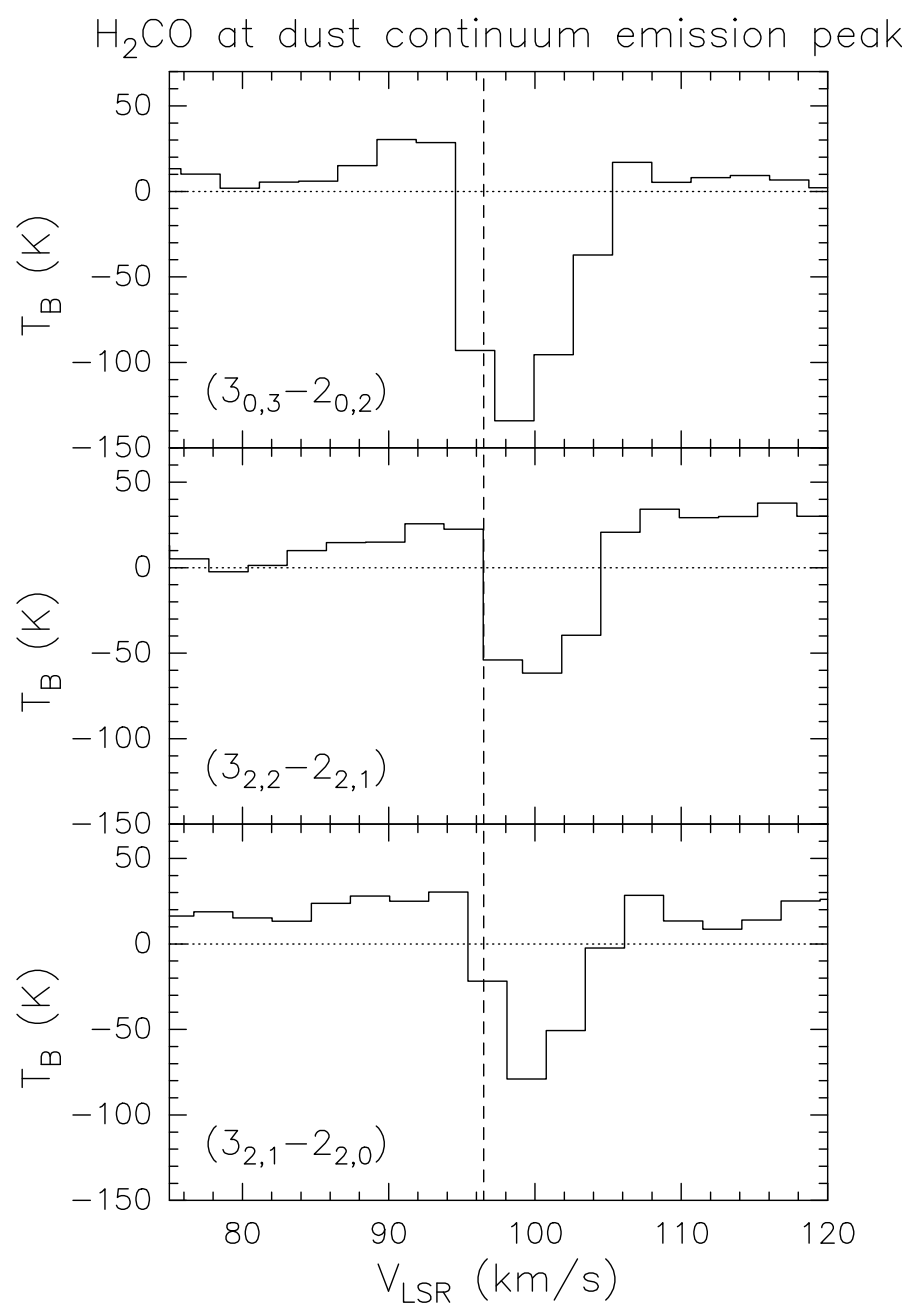

Fig. 4. $\mathrm{H}_{2} \mathrm{CO}\left(3_{0,3}-2_{0,2}\right),\left(3_{2,2}-2_{2,1}\right)$, and $\left(3_{2,1}-2_{2,0}\right)$ spectra at the dust continuum emission peak. The vertical dashed line indicates the systemic LSR velocity of $96.5 \mathrm{~km} \mathrm{~s}^{-1}$.

expects the highest energy transitions to be optically thinner and trace material closer to the central (proto)star(s). As shown in Figs. A.2 and A.3, the integrated emission of all species (methyl cyanide and isotopologues, and methyl formate) traces a ring-like structure with the dust continuum emission peak and the two free-free sources located at the central dip. This morphology is better seen in the lowest energy transitions such as $\mathrm{CH}_{3} \mathrm{CN} K=2\left(E_{\text {up }}=97 \mathrm{~K}\right)$ or $\mathrm{CH}_{3} \mathrm{OCHO}\left(19_{4,16}-18_{4,15}\right) \mathrm{A}\left(E_{\text {up }}\right.$ $=123 \mathrm{~K})$, and is still visible in transitions with energies as high as $778 \mathrm{~K}$ (e.g., $\left.\mathrm{CH}_{3} \mathrm{CN} K, l=6,1 v_{8}=1\right)$. For this vibrationally excited transition, although the emission still decreases toward the central region, the dip is less pronounced. This ring-like morphology in $\mathrm{CH}_{3} \mathrm{CN}$ had already been observed by Beltrán et al. (2005) and Cesaroni et al. (2011) with the IRAM PdBI and SMA interferometers with an angular resolution of $\sim 1^{\prime \prime}$. These studies suggest that the decrease of the $\mathrm{CH}_{3} \mathrm{CN}$ emission might be produced by self-absorption due to the high opacities in the central region of the core, combined with a temperature gradient, although none of the $\mathrm{CH}_{3} \mathrm{CN}$ lines showed evidence of selfabsorption or inverse P-Cygni profiles. As one can see in Fig. 3, the situation changes when the core is observed with an angular resolution of $\sim 0$.' 2 because the $\mathrm{CH}_{3} \mathrm{CN}$ emission toward the dust continuum emission peak clearly shows red-shifted absorption. Inverse P-Cygni profiles had been previously detected by Girart et al. (2009) in low energy lines such as $C^{34} S(7-6)$, and although
Table 2. Best fit model parameters for the $\mathrm{CH}_{3} \mathrm{OCHO} v=0$ and $v_{t}=1$ transitions for the emission azimuthally averaged at different rings.

\begin{tabular}{cccc}
\hline \hline $\begin{array}{c}R \\
(\operatorname{arcsec})\end{array}$ & $\begin{array}{c}R \\
(\mathrm{au})\end{array}$ & $\begin{array}{c}T_{\mathrm{ex}} \\
(\mathrm{K})\end{array}$ & $\begin{array}{c}N_{\mathrm{CH}_{3} \mathrm{OCHO}} \\
\left(10^{17} \mathrm{~cm}^{-2}\right)\end{array}$ \\
\hline 0.22 & 1740 & $436 \pm 20$ & $20^{a}$ \\
0.44 & 3480 & $343 \pm 29$ & $12.6 \pm 0.8$ \\
0.66 & 5200 & $233 \pm 16$ & $7.9 \pm 0.5$ \\
0.88 & 7000 & $182 \pm 11$ & $4.0 \pm 0.2$ \\
1.10 & 8700 & $136 \pm 8$ & $1.45 \pm 0.05$ \\
1.32 & 10430 & $110 \pm 10$ & $0.48 \pm 0.03$ \\
1.54 & 12170 & $114 \pm 16$ & $0.16 \pm 0.014$ \\
\hline
\end{tabular}

Notes. ${ }^{(a)}$ This value has been fixed to the value obtained by extrapolating a linear fit from the $R=0 . ' 44$ and $0 . ' 66$ rings. If both $T_{\mathrm{ex}}$ and $N_{\mathrm{CH}_{3} \mathrm{OCHO}}$ are left as free parameters, the algorithm gives an unrealistic high excitation temperature of $>1000 \mathrm{~K}$ for the $R=0$.' 22 ring.

less evident, also in $\mathrm{CH}_{3} \mathrm{OH}$ and isotopologues with SMA 1" angular resolution observations. This is the first time that the red-shifted absorption in $\mathrm{G} 31$ has been observed in $\mathrm{CH}_{3} \mathrm{CN}$ and isotopologues.

The red-shifted absorption is also clearly detected in $\mathrm{H}_{2} \mathrm{CO}$ $\left(3_{0,3}-2_{0,2}\right),\left(3_{2,2}-2_{2,1}\right)$, and $\left(3_{2,1}-2_{2,0}\right)$, which have upper level energies of 21 and $68 \mathrm{~K}$ (Fig. 4). The absorption is much deeper in $\mathrm{H}_{2} \mathrm{CO}$ than in $\mathrm{CH}_{3} \mathrm{CN}$ : the brightness temperature $T_{\mathrm{B}}$ is $-134 \mathrm{~K}$ for $\mathrm{H}_{2} \mathrm{CO}\left(3_{0,3}-2_{0,2}\right)$ and $-40 \mathrm{~K}$ for $\mathrm{CH}_{3} \mathrm{CN} K=2$.

\subsection{2. $\mathrm{SiO}$ emission}

The molecular outflows associated with G31 have been studied in CO and ${ }^{13} \mathrm{CO}$ by Olmi et al. (1996) and Cesaroni et al. (2011). The $\mathrm{CO}$ emission is very complex and reveals the presence of at least two molecular outflows in the region, one in an eastwest $(\mathrm{E}-\mathrm{W})$ direction and the other one in an almost north-south $(\mathrm{N}-\mathrm{S})$ direction. To study the outflows on scales comparable to the HMC, the ALMA correlator was set up to cover the $\mathrm{SiO}$ emission, a well-known shock tracer. As seen in Figs. 2 and A.1, the $\mathrm{SiO}(5-4)$ emission has been clearly detected toward both the Main and the NE core. The spectral profiles of $\mathrm{SiO}$ at different positions in the region clearly show broad wings typical of shocked material (Fig. 5). As seen in this figure, the $\mathrm{SiO}$ profile at the dust continuum emission peak is also affected by absorption. The $\mathrm{SiO}$ emission averaged on the blue-shifted and red-shifted wings reveals the presence of several bipolar outflows in the region. In Fig. 6, we have indicated the axes of the possible outflows associated with the core. The directions have been roughly estimated by joining the blue-shifted peaks with what we believe to be the corresponding red-shifted peaks. Another possible interpretation of the blue and red-shifted emission in Fig. 6 is that the $\mathrm{SiO}$ line is tracing a single, wide-angle bipolar outflow oriented NE-SW. In this scenario, the two lobes would be heavily resolved out by the interferometer and we detect only their borders. However, we tend to exclude this possibility, because such a prominent outflow should be clearly seen in single-dish maps of the region, in contrast with the results obtained by Cesaroni et al. (2011). These authors imaged the ${ }^{12} \mathrm{CO}$ and ${ }^{13} \mathrm{CO}(2-1)$ line emission toward the G31 region with the IRAM 30-m telescope, and find no obvious evidence of a bipolar outflow on scales $>5^{\prime \prime}(>0.2 \mathrm{pc})$.

The most clear bipolar outflow is that oriented in the E-W direction, whose geometrical center is displaced $\sim 0{ }^{\prime} 6$ ( $\sim 4700 \mathrm{au})$ to the south of the dust continuum emission peak and 

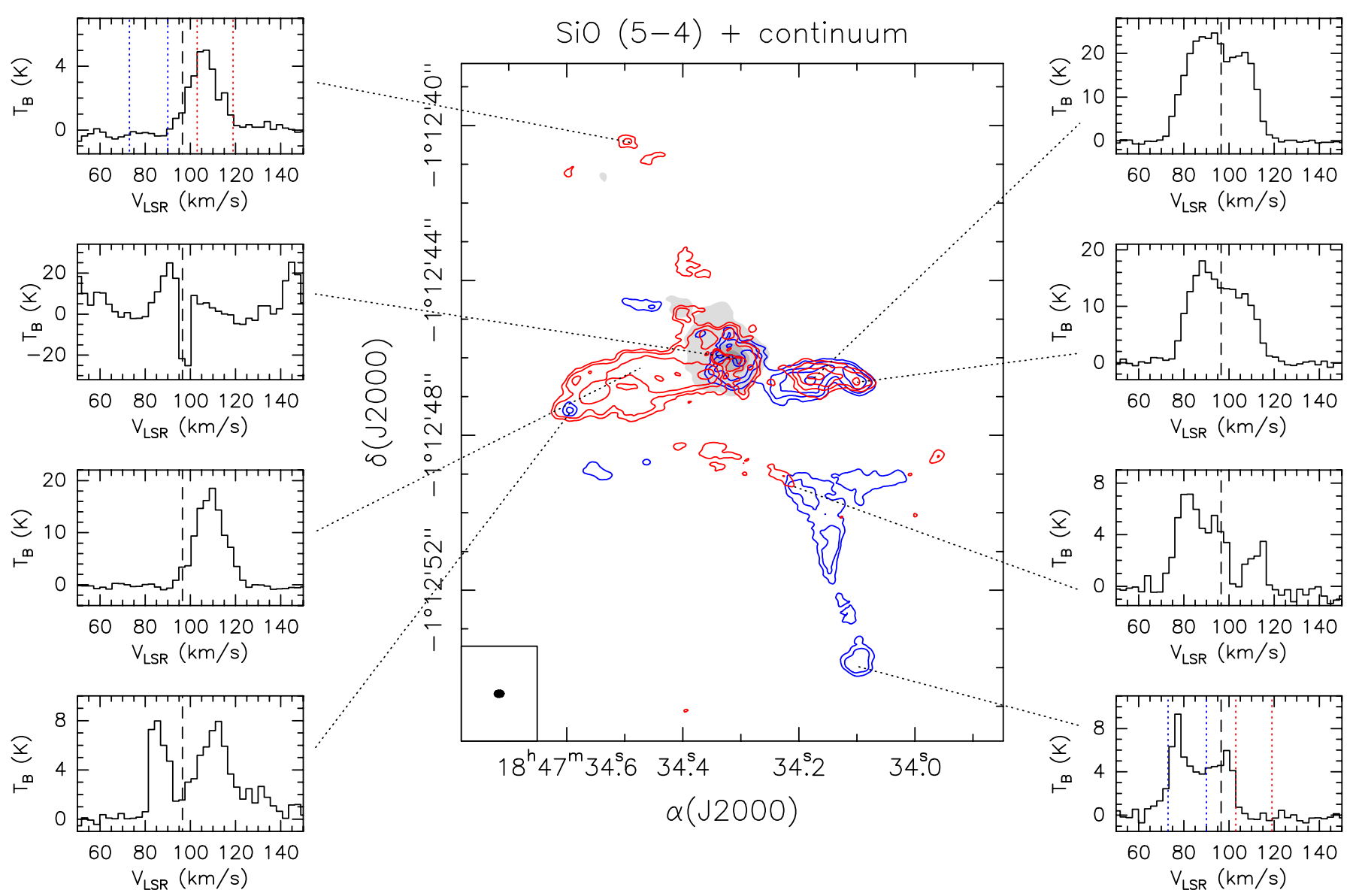

Fig. 5. Middle panel: overlay of the $217 \mathrm{GHz}$ continuum emission (grayscale) on the blue-shifted (blue contours) and red-shifted (red contours) $\mathrm{SiO}(5-4)$ averaged emission. The blue-shifted emission has been averaged over the $(73,90) \mathrm{km} \mathrm{s}^{-1}$ velocity interval and the red-shifted emission over the $(103,119) \mathrm{km} \mathrm{s}^{-1}$ one and are indicated in blue and red dotted vertical lines in the top left and lower right spectra. Contour levels are 3, 6 , 12 , and 24 times $1 \sigma$, where $1 \sigma$ is $1.1 \mathrm{mJy} \mathrm{beam}^{-1}$. Grayscale contours for the continuum emission range from 7.5 to $232.5 \mathrm{mJy} \mathrm{beam}^{-1}$ in steps of $90 \mathrm{mJy}_{\text {beam }}{ }^{-1}$. The synthesized beam is shown in the lower left-hand corner. Left and right panels: SiO (5-4) spectra toward selected positions in the G31 core. The vertical dashed line indicates the systemic LSR velocity of $96.5 \mathrm{~km} \mathrm{~s}^{-1}$.

free-free sources (see Fig. 6). We note that the eastern red-shifted lobe of this outflow could be contaminated by red-shifted emission from another outflow in the region oriented NE-SW (see below). This could explain why the red lobe of the E-W outflow appears slightly bent toward south. The extent of this E-W outflow is $\sim 8$ ". $5(\sim 0.33 \mathrm{pc})$. The fact that the blue-shifted and redshifted emissions overlap in the lobes, especially in the western one, suggests that the outflow lies close to the plane of the sky.

Another bipolar outflow is elongated in an almost $\mathrm{N}-\mathrm{S}$ direction, with red-shifted and blue-shifted knots located 7 " $-8^{\prime \prime}$ from the center of the core. The extent of this bipolar outflow is $\sim 15^{\prime \prime}(\sim 0.58 \mathrm{pc})$. This $\mathrm{N}-\mathrm{S} \mathrm{SiO}$ outflow is also visible in the ${ }^{12} \mathrm{CO}(2-1)$ maps of Cesaroni et al. (2011). The outflow extends further north in $\mathrm{SiO}$ than in $\mathrm{CO}$, probably due to the lower sensitivity of the SMA CO observations. If one traces a line from the southernmost blue-shifted knot to the northernmost red-shifted knot, the outflow seems to cross the center of the Main core, which suggests that it could be driven by one of the free-free sources. In addition, as seen in Fig. 6, this outflow could be associated with the water maser jet observed by Moscadelli et al. (2013). The analysis of the water maser dynamics by Moscadelli et al. (2013) demonstrated clearly that the water masers trace expansion, i.e., outflow(s) in the region, while the methanol and hydroxyl maser dynamics are still not fully understood.
Through inspection of the $\mathrm{SiO}$ emission, one finds a possible third outflow in a NE-SW direction (Fig. 6). This outflow is clearly not associated with the Main core and it could be associated with a southern core not detected in our observations, possibly due to limitation in the dynamical range. The $3 \sigma$ level of the dust continuum emission is $4.5 \mathrm{mJy} \mathrm{beam}^{-1}$, which is $\sim 53$ times lower than the peak emission of the Main core. This third outflow is also visible in the ${ }^{12} \mathrm{CO}$ channel maps of Cesaroni et al. (2011). As seen in the CO channel maps and in the $\mathrm{SiO}$ averaged emission map, the red-shifted NE lobe extends to the north up to the eastern red-shifted lobe of the E-W bipolar outflow.

\section{Analysis}

\subsection{Fitting the spectra}

The high sensitivity of the ALMA observations has allowed us to detect the emission of several transitions of high-density tracers, such as methyl cyanide and methyl formate, with different upper level energies. Given the high density of the core, one could derive the gas temperature and column density by fitting the lines of these molecules with a simple model assuming local thermodynamic equilibrium (LTE) conditions. Application of this method to all the spectra observed over the core would provide 

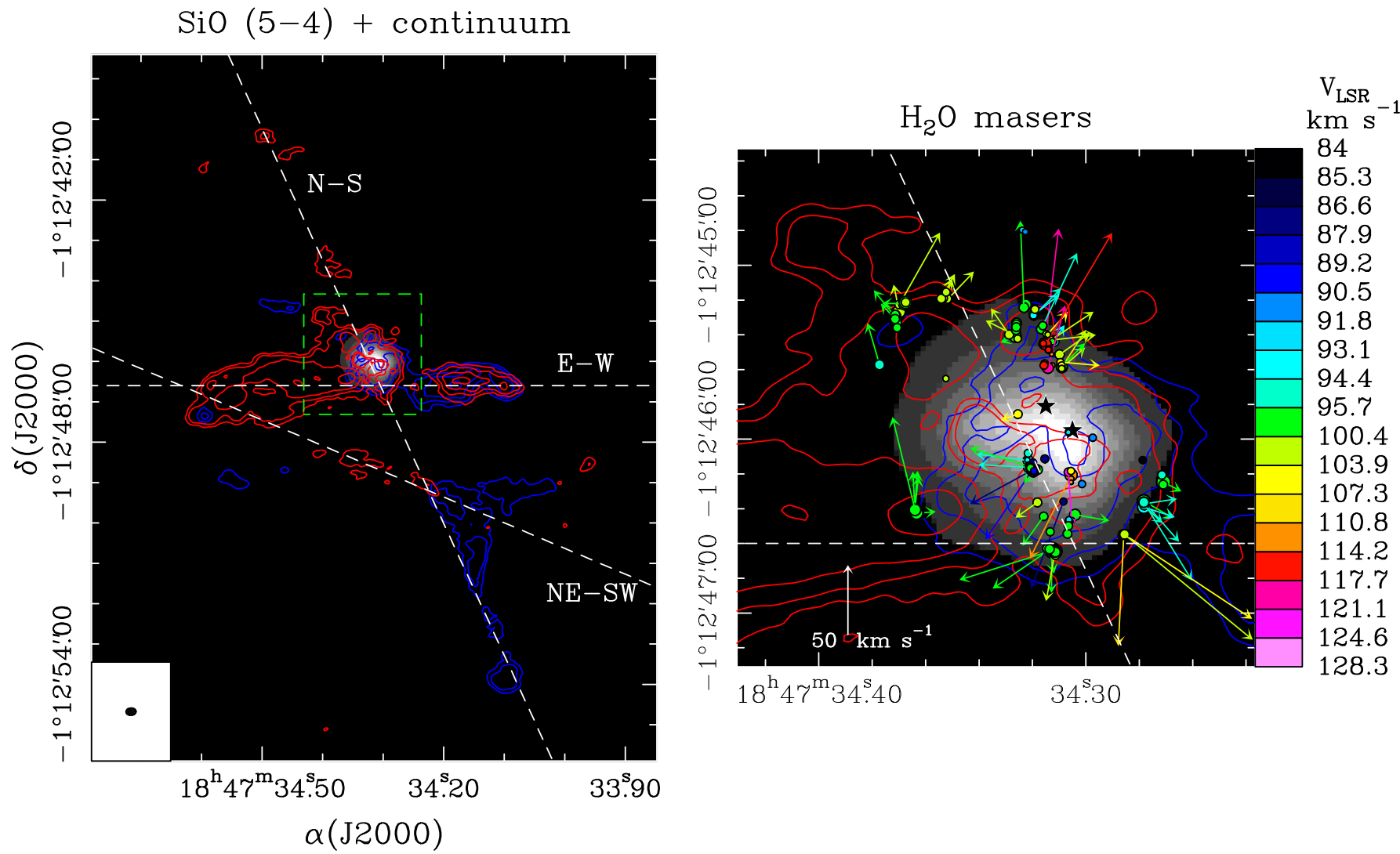

Fig. 6. Left panel: same as Fig. 5. The white dashed lines indicate the direction of the three possible outflows in G31, and the green dashed box indicates the area plotted in the close-up panel. Grayscale levels for the continuum are 10, 20, 30, 40, 50, 60, 60, 70, 80, and 90\% of the peak. Right panel: close-up of the central region. Colored circles mark the position of $\mathrm{H}_{2} \mathrm{O}$ masers while colored vectors indicate the direction and the amplitude of the proper motions (Moscadelli et al. 2013). The white vector in the bottom left corner indicates the amplitude scale of proper motions in kilometer per second. The black stars indicate the positions of the two free-free continuum sources detected by Cesaroni et al. (2010).

us with maps of excitation temperature, $T_{\mathrm{ex}}$, and column density, $N_{\text {tot }}$. While software such as XCLASS (Möller et al. 2017) can automatically perform this fit over large regions corresponding to many spectra, in the case of G31 the situation is more complicated and requires an ad hoc solution. In fact, most line profiles toward the central region of the core are severely affected by deep absorption (see Fig. 3), which calls for manual fitting to achieve convergence. In order to simplify the problem, we have assumed spherical symmetry for the G31 core, consistent with its roundish appearance. The core center has been chosen to be the position of the continuum peak and we have computed mean spectra over circular concentric annuli of width equal to that of the synthesized beam $\left.(\sim 0)^{\prime} 22\right)$, up to a maximum radius of 1".54, beyond which most of the lines are not detected (see Figs. A.2 and A.3). In this way, by fitting the mean spectra, we have obtained $T_{\text {ex }}$ and $N_{\text {tot }}$ as a function of distance from the core center.

In this work, we have used two different programs to fit the lines: MADCUBA ${ }^{4}$ (Martín et al. in prep.; see also Rivilla et al. 2016) and XCLASS (Möller et al. 2017). Both programs take line blending and optical depth effects into account and in both cases, the emission is described with 5 parameters that can be fixed or left free: the source size $\theta$, the excitation temperature $T_{\text {ex }}$, the column density of the molecule, $N_{\text {tot }}$, the line

4 Madrid Data Cube Analysis on ImageJ is a software developed in the Center of Astrobiology (Madrid, INTA-CSIC) to visualize and analyze single spectra and datacubes (Martín et al., in prep.). width, $\Delta v$, and the peak velocity of the emission $v$. The continuum emission can be described as a modified blackbody. In general, the outputs of the two codes are consistent. However, in specific cases one of the two algorithms appears to achieve better convergence than the other. In particular, to fit the spectra at the core center, where both emission and absorption components are detected, XCLASS is preferred to MADCUBA. The latter package models the core and foreground layers as independent components that do not interact with each other radiatively, whereas in XCLASS, the core contribution is simulated as an additional continuum background (Möller et al. 2017). This approach resembles our scenario where all the components along the line of sight interact radiatively. On the other hand, when estimating the temperature and column density profiles across the core (see next section) by fitting a single component in emission, we have used MADCUBA because the fitting algorithm converges faster than XCLASS. It should be noted that the $\mathrm{CH}_{3} \mathrm{OCHO}$ emission toward the center, for which the absorption is strongly affecting the profiles of the emission lines, has not been fitted because one needs at least two components. The fitting of the central region is discussed in Sect. 5.2.2.

\subsection{Temperature and density profiles}

To estimate the physical parameters across the core, we fitted the ground state rotational transitions of methyl formate $(v=0)$ and those in the first torsionally excited state $\left(v_{t}=1\right)$. The choice 
of $\mathrm{CH}_{3} \mathrm{OCHO}$ instead of $\mathrm{CH}_{3} \mathrm{CN}$ is based on the fact that the $\mathrm{CH}_{3} \mathrm{CN}$ spectra closer to the central position $\left(R \leq 0\right.$.' $\left.^{\prime} 66\right)$ cannot be properly fitted because of the high opacity of the ground state transitions. In addition, as already mentioned, we have detected $\sim 40 \mathrm{CH}_{3} \mathrm{OCHO}$ transitions (including both $v=0$ and $v_{t}=1$ ) that are either unblended or only slightly blended with other lines, whereas only few unblended $\mathrm{CH}_{3} \mathrm{CN}$ transitions (including $v=0, v_{8}=1$, and isotopologues) could be identified. This allows us to better constrain the fit parameters. The fitting strategy we adopted in MADCUBA was to fix the line width to a value that provides a good visual fit, and the source size, that we assumed to be larger than the beam size. For the dust continuum temperature at each annulus, we assumed the value of the azimuthally averaged brightness temperature of the dust emission. Table 2 shows the best-fit $T_{\mathrm{ex}}$ and $N_{\mathrm{CH}_{3} \mathrm{OCHO}}$ values at each radius. A successful fit to the spectra for the innermost radius was not achieved due to the strong absorption, which affects the profiles and prevents the algorithm from converging. The fit of the $R=0.22$ ring gives an unrealistically high excitation temperature of $>1000 \mathrm{~K}$ if both $T_{\text {ex }}$ and $N_{\mathrm{CH}_{3} \mathrm{OCHO}}$ are left as free parameters. Therefore, we fixed the column density to $2 \times 10^{18} \mathrm{~cm}^{-2}$, the value obtained from linear extrapolation of the column densities at the two adjacent inner rings.

Figure 7 shows the temperature and column density, estimated by fitting the $\mathrm{CH}_{3} \mathrm{OCHO} v=0$ and $v_{t}=1$ emission, as a function of radius, $R$, and the least-square linear fits. The best fit to the $\log \left(T_{\text {ex }}\right)$ vs. $\log (R)$ relation is:

$\log \left(T_{\text {ex }} / \mathrm{K}\right)=(2.19 \pm 0.02)-(0.77 \pm 0.07) \log (R / \operatorname{arcsec})$.

The fit implies that $T_{\mathrm{ex}}$ is proportional to $R^{-0.77}$. This temperature profile is much steeper than the $R^{-0.4}$ profile found at the outer parts of envelopes surrounding massive YSOs by van der Tak et al. (2000), but is consistent with the profiles of hot molecular cores modeled by Nomura \& Millar (2004) and Osorio et al. (2009). Alternatively, the $T_{\text {ex }}$ profile would also be consistent with those estimated for passive, geometrically flat, irradiated dust disks (Adams \& Shu 1986) or steadily accreting, thin disks (Shakura \& Sunyaev 1973), which have power-law exponents of -0.75 .

As shown in Fig. $7 \mathrm{~b}$, the $\mathrm{CH}_{3} \mathrm{OCHO}$ column density profile is best fitted by a broken power law. The best fit to the $\log \left(N_{\mathrm{CH}_{3} \mathrm{OCHO}}\right)$ vs. $\log (R)$ relation is:

$$
\begin{aligned}
& \log \left(N_{\mathrm{CH}_{3} \mathrm{OCHO}} / \mathrm{cm}^{-2}\right)= \\
& \left\{\begin{array}{cc}
(17.17 \pm 0.05)-(4.63 \pm 0.41) \log (R / \operatorname{arcsec}) & R>0.66^{\prime \prime} \\
(17.77 \pm 0.06)-(0.82 \pm 0.12) \log (R / \operatorname{arcsec}) & R \leq 0.66^{\prime \prime}
\end{array}\right.
\end{aligned}
$$

In the inner part of the core, the column density is proportional to $R^{-0.8}$, much flatter than the outer part of the core, which shows a very steep profile $R^{-4.6}$. The fact that the $N_{\mathrm{CH}_{3} \mathrm{OCHO}}$ profile flattens toward the center of the core is likely an effect of absorption, which is affecting the $\mathrm{CH}_{3} \mathrm{OCHO}$ line profiles in the inner rings $(R<5000 \mathrm{au})$. To study whether the drastic drop of $\mathrm{CH}_{3} \mathrm{OCHO}$ column density is due to a decrease of the $\mathrm{CH}_{3} \mathrm{OCHO}$ abundance or is produced by a drop of the gas density toward the center of the core, we estimated the $\mathrm{H}_{2}$ column density from the dust continuum emission azimuthally averaged over the same concentric rings as the $\mathrm{CH}_{3} \mathrm{OCHO}$ emission. We assumed that the dust temperature is equal to the excitation temperature estimated for $\mathrm{CH}_{3} \mathrm{OCHO}$ at each ring (see Table 2). Figure 7c shows $N_{\mathrm{H}_{2}}$ as a function of $R$. As seen in the figure, $\log \left(N_{\mathrm{H}_{2}}\right)$ follows a linear correlation with $\log (R)$. The best fit is:

$\log \left(N_{\mathrm{H}_{2}} / \mathrm{cm}^{-2}\right)=(24.01 \pm 0.02)-(1.15 \pm 0.08) \log (R / \operatorname{arcsec})$.

The $\mathrm{H}_{2}$ column density seems to be proportional to $R^{-1.2}$, and the profile does not show an abrupt change of slope at $R>$ 5000 au like that observed in $\mathrm{CH}_{3} \mathrm{OCHO}$. The dust emission at inner radii is optically thick, as indicated by the high brightness temperature of the continuum peak $(\sim 132 \mathrm{~K})$ being comparable to the gas (and dust) temperature $(\gtrsim 150 \mathrm{~K})$. Therefore, the $\mathrm{H}_{2}$ column density values should be taken as lower limits. However, the slope of the power-law distribution is very steep $(-1.15)$, and therefore, the correction due to the large continuum opacity should likely not affect the estimated column density dramatically. Otherwise, the volume density profile would be extremely steep ( $\propto r^{-n}$ with $n>2.2$ ), difficult to justify in (roughly) spherical geometry either for a static envelope or an accretion flow (e.g., van der Tak et al. 2000). This suggests that the drop seen in $\mathrm{CH}_{3} \mathrm{OCHO}$ column density is due to a decrease of its abundance, as shown by the $\left[N_{\mathrm{CH}_{3} \mathrm{OCHO}} / N_{\mathrm{H}_{2}}\right]$ ratio in Fig. $7 \mathrm{~d}$. While for $R<5000$ au the $\mathrm{CH}_{3} \mathrm{OCHO}$ abundance is almost constant with a value of $\sim 4 \times 10^{-7}$, for $R>5000$ au the abundance is proportional to $R^{-3.2}$ and drops almost an order of magnitude at the outer rings. An even more drastic decrease of the abundance at the outer part of the core is observed for $\mathrm{CH}_{3} \mathrm{CN}$. Unfortunately, for this species, it has not been possible to fit the emission for the rings with $R<7000 \mathrm{au}$, so it is not possible to investigate whether the abundance is constant at the center of the core. The best least-square linear fits to the $\mathrm{CH}_{3} \mathrm{OCHO}$ abundance are:

$$
\begin{aligned}
& \log \left[N_{\mathrm{CH}_{3} \mathrm{OCHO}} / N_{\mathrm{H}_{2}}\right]= \\
& \left\{\begin{array}{cc}
-(6.85 \pm 0.05)-(3.23 \pm 0.36) \log (R / \operatorname{arcsec}) & R>0.66^{\prime \prime} \\
-(6.35 \pm 0.01)+(0.08 \pm 0.01) \log (R / \operatorname{arcsec}) & R \leq 0.66^{\prime \prime}
\end{array}\right.
\end{aligned}
$$

The rich chemistry of hot molecular cores, in particular of complex molecules such as $\mathrm{CH}_{3} \mathrm{OCHO}$, is the result of the evaporation of dust grain mantles. One possibility to explain the drastic drop of the $\mathrm{CH}_{3} \mathrm{OCHO}$ abundance could be that the temperature is not high enough to desorb $\mathrm{CH}_{3} \mathrm{OCHO}$ from the dust grains. However, Burke et al. (2015) have conducted laboratory experiments and estimated that $\mathrm{CH}_{3} \mathrm{OCHO}$ desorbs at a temperature of about $70 \mathrm{~K}$, which is much lower than the excitation temperatures estimated in the outer rings of G31 (>110 K). Therefore, the only plausible explanation for the abundance drop observed in the $\mathrm{G} 31$ core is that $\mathrm{CH}_{3} \mathrm{OCHO}$ is concentrated in the regions closer to the central protostar.

\subsection{Mass estimates}

Previous mass estimates of G31 range from $\sim 500$ to $1700 \mathrm{M}_{\odot}$ (Beltrán et al. 2004; Girart et al. 2009; Cesaroni et al. 2011). These masses have been derived from the dust continuum emission, assuming a single value of the temperature. The large discrepancy is mainly due to the different dust absorption coefficient used by the different authors. Because we have a better knowledge of the physical properties of the core, having derived the temperature and density distribution of the core (see previous section), we can improve the mass estimate of G31. To do this, we estimated the mass of the core, $M_{c}$, assuming that the dust emission is optically thin and in the Rayleigh-Jeans 

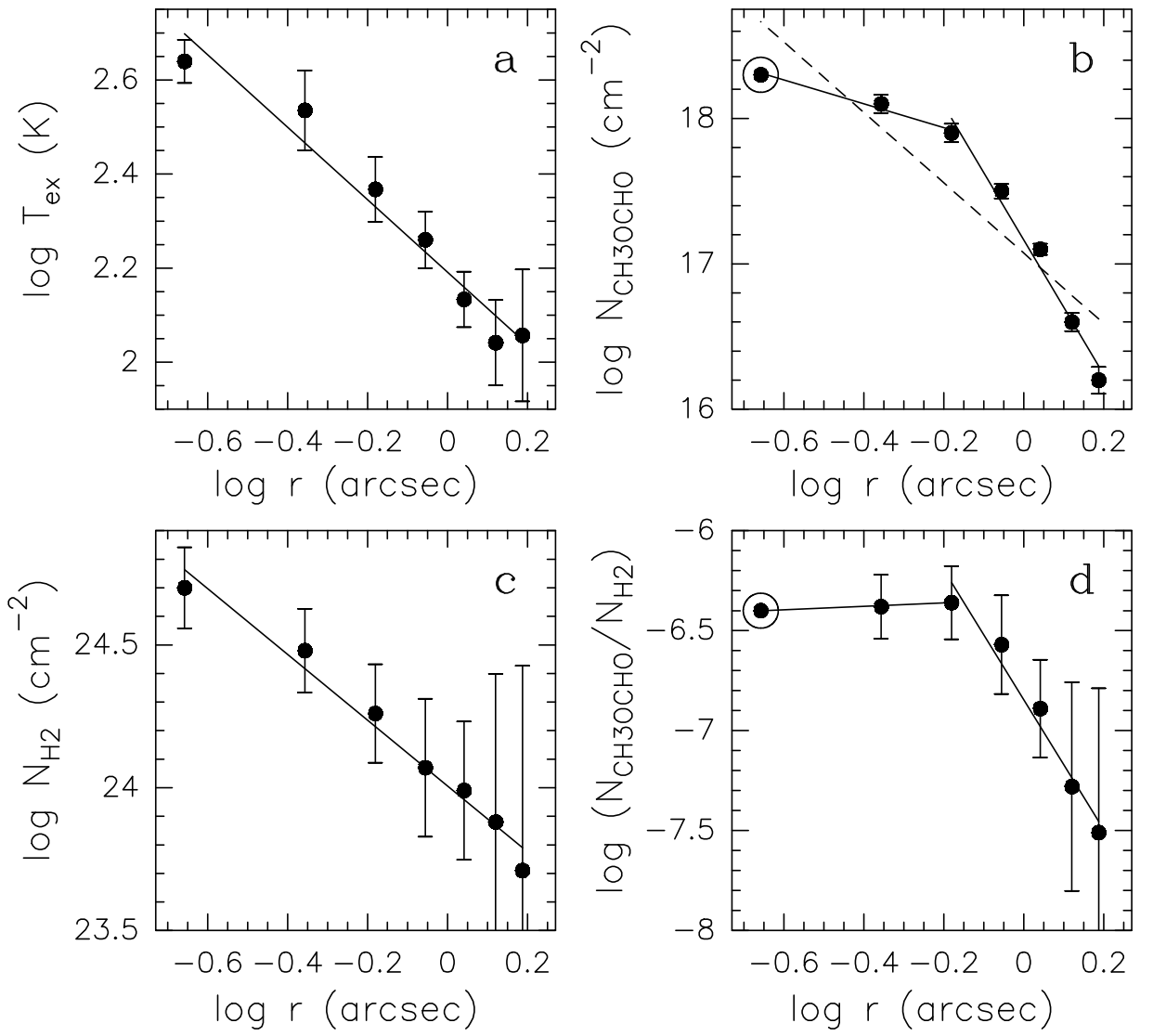

Fig. 7. Panel $a$ : Excitation temperature, panel b: $\mathrm{CH}_{3} \mathrm{OCHO}$ column density, panel $c: \mathrm{H}_{2}$ column density, and panel $d:\left[N_{\mathrm{CH}_{3} \mathrm{OCHO}} / N_{\mathrm{H}_{2}}\right]$ ratio as a function of the radius. $T_{\text {ex }}$ and $N_{\mathrm{CH}_{3} \mathrm{OCHO}}$ have been estimated by fitting the $\mathrm{CH}_{3} \mathrm{OCHO}$ $v=0$ and $v_{t}=1$ emission azimuthally averaged over different rings (see Sect. 4.2), while $N_{\mathrm{H}_{2}}$ has been estimated from the azimuthally averaged dust emission. The $\mathrm{CH}_{3} \mathrm{OCHO}$ column density at a radius of 0.22 (marked with a circle) has been calculated by extrapolating a linear fit from the $R=0.44$ and $0{ }^{\prime} 66$ rings. The solid lines in panels $a$ and $c$ and the dashed one in panel $b$ show the least square linear fits to all the points. The solid lines in panels $b$ and $d$ show the least square linear fits to the inner 3 points and to the outer 5 points in each panel. regime (in fact, $\left[\frac{v}{\mathrm{GHz}}\right] \ll 20\left[\frac{T_{\mathrm{ex}}}{\mathrm{K}}\right]$ ), and that the density and temperature of the core follow power-law distributions, $\rho \propto r^{-n}$ and $T \propto r^{-q}$. By integrating the density distribution over the volume, one finds that $M_{c}=\frac{4 \pi}{3-n} \rho_{c} r_{c}^{3}$, where $\rho_{c}$ is the density at the radius of the core, $r_{c}$. Following Eqs. (1) and (2) of Beltrán et al. (2002), the density at the radius of the core can be written as

$\rho_{c}=\frac{1}{4 \pi \sqrt{\pi}} \frac{\Gamma[(n+q) / 2]}{\Gamma[(n+q-1) / 2]} \frac{3-n-q}{r_{c}^{3}} \frac{c^{2}}{k v^{2} T_{c} \kappa_{v}} d^{2} S_{v}$,

where $\Gamma(x)$ is the gamma function, $\kappa$ is the Boltzmann constant, $v$ is the frequency of the observations, $T_{c}$ is the temperature at the radius of the core, $\kappa_{v}$ is the dust absorption coefficient per unit mass, $d$ is the distance of the source, and $S_{v}$ is the integrated flux density over the whole core. Using this equation, $M_{c}$ can be written as

$M_{c}=\frac{1}{\sqrt{\pi}} \frac{\Gamma[(n+q) / 2]}{\Gamma[(n+q-1) / 2]} \frac{3-n-q}{3-n} \frac{c^{2}}{k v^{2} T_{c} \kappa_{v}} d^{2} S_{v}$.

We estimated $\rho_{c}$ and $M_{c}$ for the G31 Main core, for a radius $r_{c}$ of 8500 au $\left(\sim 1^{\prime \prime} .076\right)$, which is the deconvolved radius of the dusty core at the $5 \sigma$ emission level. For this purpose, we adopted $q=0.77$ and $T_{c}=146 \mathrm{~K}$ (see Eq. (1)), $v=217 \mathrm{GHz}$, $k_{v}=0.008 \mathrm{~cm}^{2} \mathrm{~g}^{-1}$ (Ossenkopf \& Henning 1994 and a gas-todust mass ratio of 100 ), and $S_{v}=3.10 \mathrm{Jy}$ (see Table 1). The $\mathrm{H}_{2}$ column density profile (Eq. (3)) implies a density power-law exponent $n \sim 2.2$. On the other hand, the red-shifted absorption observed in several tracers suggests that the core might be undergoing free-fall collapse (see Sect. 3.2.1 and 5.2) with $n \sim 1.5$. Therefore, we assumed an intermediate value of $n=2$. This leads to $\rho_{c}=9 \times 10^{-18} \mathrm{~g} \mathrm{~cm}^{-3}$, corresponding to a volume density of $\sim 5.4 \times 10^{6} \mathrm{~cm}^{-3}$, and $M_{c} \simeq 120 M_{\odot}$. Because the mass has been estimated assuming that the dust emission is optically thin, the value derived has to be taken as a lower limit in case this condition is not satisfied.

\section{Discussion}

\subsection{The NE-SW velocity gradient}

Figure A.2 shows the $217 \mathrm{GHz}$ continuum emission overlaid on the integrated intensity (moment 0 ), line velocity (moment 1 ), and velocity dispersion (moment 2) maps of different transitions of $\mathrm{CH}_{3} \mathrm{CN}$ (ground state and vibrationally excited) and isotopologues. These transitions have been selected to trace emission with upper level energies ranging from 97 to $778 \mathrm{~K}$. The emission of methyl cyanide shows a clear velocity gradient along the NE-SW direction regardless of the upper level energy of the transition. The position angle of the velocity gradient is $\sim 68^{\circ}$ and is roughly consistent with that of the HMC dust continuum emission, PA $\sim 63^{\circ}$ (see Sect. 3.1 ). The same velocity gradient is observed in $\mathrm{CH}_{3} \mathrm{OCHO}$ (Fig. A.3). This velocity gradient, already observed in $\mathrm{CH}_{3} \mathrm{CN}$ by Beltrán et al. (2004) and Cesaroni et al. (2011) and in $\mathrm{CH}_{3} \mathrm{OH}$ by Girart et al. (2009) has been interpreted as rotation of the core. The velocity gradient is $\sim 50 \mathrm{~km} \mathrm{~s}^{-1} \mathrm{pc}^{-1}$ for $\mathrm{CH}_{3} \mathrm{CN}$ and $\mathrm{CH}_{3}{ }^{13} \mathrm{CN} \mathrm{K}=2$ and increases for the higher energy transitions, being $\sim 87 \mathrm{~km} \mathrm{~s}^{-1} \mathrm{pc}^{-1}$ for $K=$ 8 and $\sim 107 \mathrm{~km} \mathrm{~s}^{-1} \mathrm{pc}^{-1}$ for $K, l=(6,1) v_{8}=1$.

\subsubsection{Rotational spin-up}

The velocity gradient is also evident in Fig. 8, which shows the position-velocity $(\mathrm{PV})$ plots along $\mathrm{PA}=68^{\circ}$ for the same 


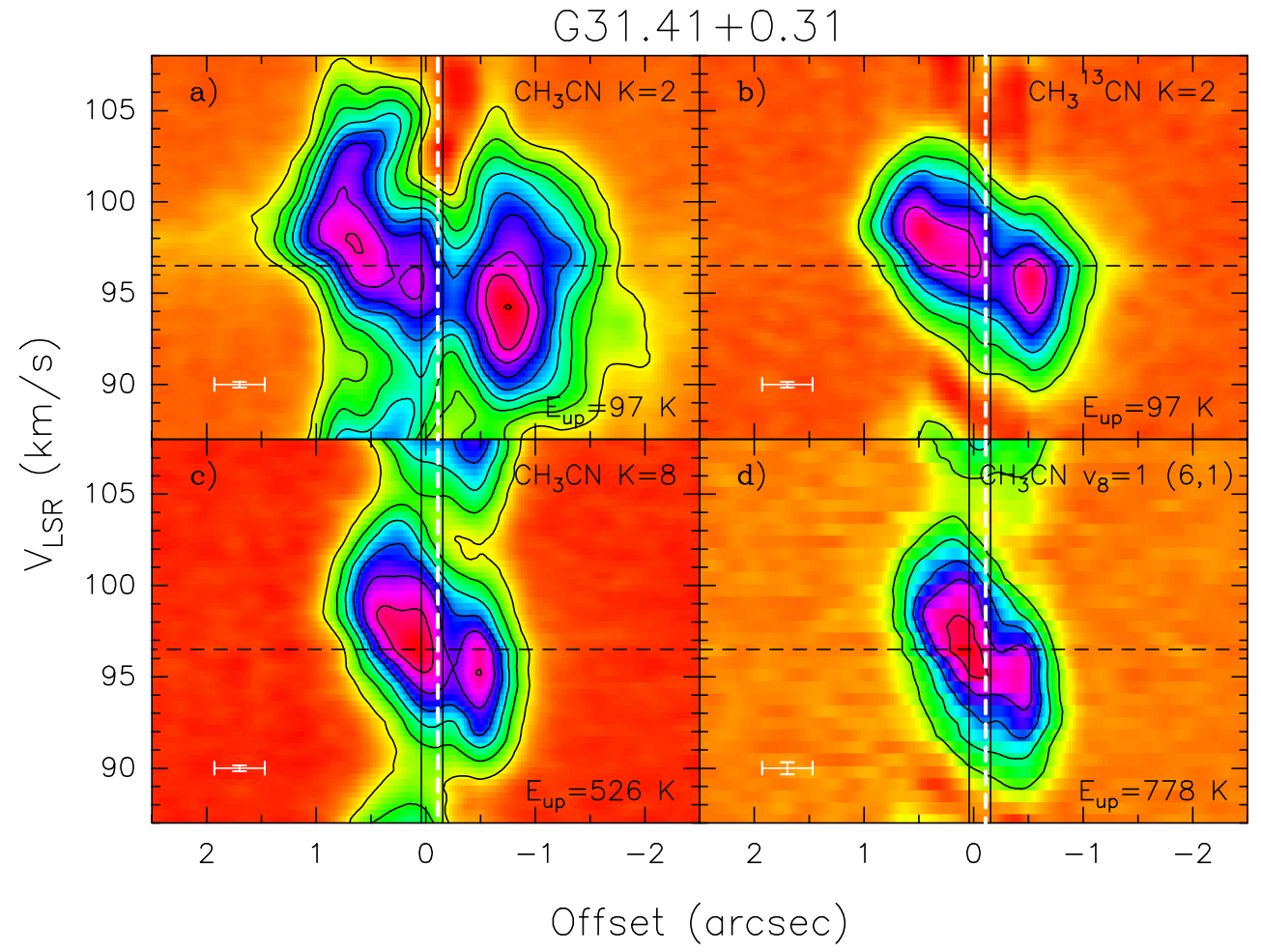

Fig. 8. Position-velocity plots along the direction with $\mathrm{PA}=68^{\circ}$ toward the $\mathrm{HMC}$ of panel a: $\mathrm{CH}_{3} \mathrm{CN} K=2$, panel b: $\mathrm{CH}_{3}{ }^{13} \mathrm{CN} K=2$, panel $c: \mathrm{CH}_{3} \mathrm{CN}$ $K=8$, and panel $d: \mathrm{CH}_{3} \mathrm{CN} K, l=$ $(6,1) v_{8}=1(12-11)$. The offsets are measured from the phase center, positive to the northeast. Contour levels range from 20 to $180 \mathrm{mJy}^{-1}$ beam $^{-1}$ in steps of $20 \mathrm{mJy}^{-1} \mathrm{bem}^{-1}$. The vertical dashed white line indicates the position of the continuum peak, while the vertical solid black lines show the position of the two compact free-free continuum sources detected by Cesaroni et al. (2010). The horizontal dashed line indicates the systemic LSR velocity. The error bars in the lower left-hand corner of the panels indicate the angular and spectral resolution of the data. The upper level energies $E_{\text {up }}$ of each transition are indicated in the lower righthand corner of the panels. transitions as in Fig. A.2. The $V_{\mathrm{LSR}}$ appears to increase linearly with distance from the core center, consistent with solid-body rotation. Figure 8 also shows the $\mathrm{CH}_{3} \mathrm{CN} K=2$ line absorption at red-shifted velocities. By comparing the different $\mathrm{PV}$ plots, one sees that the rotation seems to speed up with increasing energy of the transition. The effect is especially remarkable if one compares the PV plot of the vibrationally excited $\mathrm{CH}_{3} \mathrm{CN}(12-11)$ $K, l=(6,1) v_{8}=1\left(E_{\text {up }}=778 \mathrm{~K}\right)$ line with the PV plot of the ground-state $\mathrm{CH}_{3}{ }^{13} \mathrm{CN}(12-11) K=2\left(E_{\text {up }}=97 \mathrm{~K}\right)$ line. The former looks steeper than the latter. The $\mathrm{CH}_{3} \mathrm{CN} K=2$ transition is not considered because it is too affected by red-shifted absorption. The same steepness is also visible in the PV plot of the $K=8$ transition of $\mathrm{CH}_{3} \mathrm{CN}(12-11)\left(E_{\text {up }}=526 \mathrm{~K}\right)$ line. The PV plot of the vibrationally excited line shows emission that extends up to $\sim \pm 0$ '. 65 from the central position and has velocities ranging from 89 to $104 \mathrm{~km} \mathrm{~s}^{-1}$, while the emission in the $\mathrm{CH}_{3}{ }^{13} \mathrm{CN} K=2$ transition extends up to $\sim \pm 1^{\prime \prime}$. 1 from the center and the velocities range from 90 to $\sim 103.5 \mathrm{~km} \mathrm{~s}^{-1}$. This corresponds to slopes of $\sim 300 \mathrm{~km} \mathrm{~s}^{-1} \mathrm{pc}^{-1}$ and $\sim 160 \mathrm{~km} \mathrm{~s}^{-1} \mathrm{pc}^{-1}$, respectively. This change in slope of the PV plots of the low and high energy transitions is emphasized in Fig. 9, which shows the PV plot along the direction with $\mathrm{PA}=68^{\circ}$ of the ratio between the $\mathrm{CH}_{3} \mathrm{CN} K, l=(6,1) v_{8}=1$ (12-11) and the $\mathrm{CH}_{3}{ }^{13} \mathrm{CN} K=2$ line. The ratio is largest at small offsets and smallest at large radii. If the gradient is interpreted as rotation, this PV plot suggests that the rotation increases with increasing energy of the transition, that is, toward the core center. This is expected for conservation of angular momentum in a rotating and infalling structure. This result seems to be inconsistent with the solid-body rotation suggested by the PV in each line (see above). A possible explanation is that each line is tracing only a relatively narrow annulus of material where the gas temperature maximizes the emission from that line due to the fact that such temperature is comparable to the line upper level energy. This would explain why the projection of the rotation velocity along the line of sight increases linearly with distance form the star, mimicking solid-body rotation. At the same time, higher energy lines trace smaller annuli, closer to the star and thus rotating faster, which explains the increasing steepness of the PV plot with increasing line energy.

A scenario in which rotation spins up close to the center of the core is different from that depicted by Girart et al. (2009), who find evidence of decreasing rotation velocities toward the center of G31 and suggest that such a spin-down could be produced by magnetic braking. Girart et al. (2009) estimated the rotation velocities at the position where the dust major axis intersects the $50 \%$ contour level in the PV plots of methanol transitions with different upper level energies. On the other hand, from Fig. 3 of Girart et al. (2009), one can see that the PV plot of $\mathrm{CH}_{3} \mathrm{OH} v_{t}=2\left(7_{5}-6_{5}\right)$, which has $E_{\text {up }}>900 \mathrm{~K}$ is clearly steeper than that of $\mathrm{CH}_{3} \mathrm{OH} v_{t}=2\left(3_{3}-4_{2}\right) \mathrm{E}$, with a significantly lower energy $E_{\text {up }} \sim 60 \mathrm{~K}$, in agreement with our findings. This suggests that the discrepancy between our interpretation and that of Girart et al. is not the result of the choice of the species used to study the rotation velocity but of the method used to analyze the data. Given the uncertainty in estimating the size and corresponding velocity from the $50 \%$ contour level in a PV plot, we believe that the rotation curve obtained by Girart et al. is not to be taken as strong evidence against our interpretation of increasing rotation speed toward the center of the core.

It is also worth analyzing the velocity dispersion in the HMC. The bottom panels of Fig. A.2 show the maps of the 2nd moment of the methyl cyanide lines. For the transitions less affected by red-shifted absorption, namely those with higher energy, the velocity dispersion peaks toward the position of the dust continuum emission peak, as expected for rotation spin up. For $\mathrm{CH}_{3} \mathrm{CN}$ and $\mathrm{CH}_{3}{ }^{13} \mathrm{CN} \mathrm{K}=2$, clearly affected by absorption, the velocity dispersion increases in the directions associated with the molecular outflows in the region. 


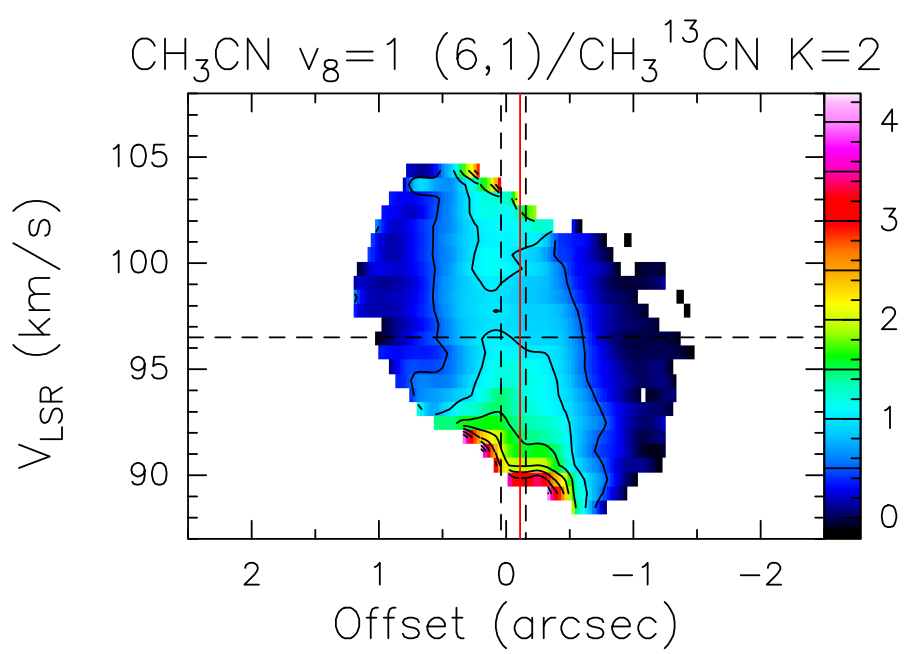

Fig. 9. Position-velocity plot along the direction with $\mathrm{PA}=68^{\circ}$ of the $\mathrm{CH}_{3} \mathrm{CN} K, l=(6,1) v_{8}=1(12-11)$ line divided by the same plot of the $\mathrm{CH}_{3}{ }^{13} \mathrm{CN} K=2$ line. The offsets are measured from the phase center, positive to the northeast. Contour levels range from 0.5 to 4 in steps of 0.5 . The vertical solid red line indicates the position of the continuum peak, while the vertical dashed black lines show the positions of the two compact free-free continuum sources detected by Cesaroni et al (2010). The horizontal dashed line indicates the systemic LSR velocity of $96.5 \mathrm{~km} \mathrm{~s}^{-1}$.

\subsubsection{Interaction with the molecular outflow}

Figure 6 shows the presence of at least three outflows in the region. It has been hypothesized that the NE-SW velocity gradient seen in high-density tracers could indeed be produced by one of these outflows (Gibb et al. 2004; Araya et al. 2008). The question was investigated by Cesaroni et al. (2011), who concluded that although the rotation scenario appeared more plausible, the outflow interpretation could not be totally discarded. With the increased angular resolution of our ALMA observations, we can now reconsider the possible relationship between the outflows in the region and the velocity gradient.

The bipolar outflow that could be interacting with the core material and producing the NE-SW gradient would be what we have called outflow E-W (Fig. 6). This outflow has been traced in ${ }^{12} \mathrm{CO}$ and ${ }^{13} \mathrm{CO}$ by Cesaroni et al. (2011) and, as seen in their lower angular resolution maps, it appears to have its geometrical center close to the center of the core. However, as seen in Fig. 10, when observed at higher angular resolution, the outflow appears clearly displaced from the center of the core and not associated with any of the embedded free-free sources nor the dust continuum peak, whereas the velocity gradient seems to be centered at the dust continuum peak. Furthermore, the direction of the outflow is significantly different from that of the velocity gradient: whereas the former is oriented in an $\mathrm{E}-\mathrm{W}$ direction, the latter, when traced in the higher energy $\mathrm{CH}_{3} \mathrm{CN} v_{8}=1$ transitions, is oriented NE-SW (see Fig. 10). This indicates that the motion of the innermost material close to the center (traced by the highest energy transitions) is not consistent with that of the bipolar outflow. Figure 10 shows the superposition of the velocity gradient traced in $\mathrm{CH}_{3} \mathrm{CN} K=2$ (top panel) and the $\mathrm{SiO}$ molecular outflow. As one can see, the $K=2$ transition traces much more extended, and possibly less dense, material than the $v_{8}=1$ transition. The line velocity (moment 1) map of $\mathrm{CH}_{3} \mathrm{CN}$ shows a red-shifted peak at $\sim 100 \mathrm{~km} \mathrm{~s}^{-1}$ and a blue-shifted peak at $\sim 92 \mathrm{~km} \mathrm{~s}^{-1}$ coincident with the lobes of the bipolar outflow and aligned in an $\mathrm{E}-\mathrm{W}$ direction, which suggests that the bipolar
Table 3. E-W outflow parameters calculated from $\mathrm{SiO}$ and $\mathrm{CH}_{3} \mathrm{CN} v_{8}=1$.

\begin{tabular}{|c|c|c|}
\hline Parameters & $\mathrm{SiO}(5-4)^{a}$ & $\begin{array}{c}\mathrm{CH}_{3} \mathrm{CN}^{b}(12-11) \\
v_{8}=1 K, l=(6,1)\end{array}$ \\
\hline$M\left(M_{\odot}\right)$ & $0.36-3.8$ & 274-7000 \\
\hline$P\left(M_{\odot} \mathrm{km} \mathrm{s}^{-1}\right)$ & $4.5-47$ & $(1-27) \times 10^{3}$ \\
\hline$E\left(L_{\odot} \mathrm{yr}\right)$ & $(0.50-5.3) \times 10^{4}$ & $(0.4-10) \times 10^{6}$ \\
\hline$\dot{M}\left(M_{\odot} \mathrm{yr}^{-1}\right)$ & $(0.60-6.3) \times 10^{-4}$ & $0.05-1.2$ \\
\hline$\dot{P}\left(M_{\odot} \mathrm{km} \mathrm{s}^{-1} \mathrm{yr}^{-1}\right)$ & $(0.75-7.8) \times 10^{-3}$ & $0.17-4.5$ \\
\hline$\dot{E}\left(L_{\odot}\right)$ & $0.83-8.8$ & $67-1667$ \\
\hline
\end{tabular}

Notes. ${ }^{(a)}$ Parameters estimated assuming a range of gas temperature of $20-50 \mathrm{~K}$ and a range of abundance relative to $\mathrm{H}_{2}$ of $10^{-8}-10^{-7}$. The blue-shifted velocity interval is $\sim(73-90) \mathrm{km} \mathrm{s}^{-1}$, and the red-shifted one $\sim(103-119) \mathrm{km} \mathrm{s}^{-1}$. ${ }^{(b)}$ Parameters estimated assuming a range of gas temperature of $100-250 \mathrm{~K}$, and an abundance relative to $\mathrm{H}_{2}$ of $10^{-8}$. The blue-shifted velocity interval is $\sim(88-95) \mathrm{km} \mathrm{s}^{-1}$, and the red-shifted one $\sim(98-105) \mathrm{km} \mathrm{s}^{-1}$.

outflow could be indeed interacting with the outer regions of the core. If the $\mathrm{CH}_{3} \mathrm{CN} \mathrm{K}=2$ emitting gas is entrained by the molecular outflow, then one would expect this gas to be tracing the high-velocity material in a similar way to $\mathrm{SiO}$. To check if this is the case, we averaged both the high-velocity $\mathrm{SiO}$ and $\mathrm{CH}_{3} \mathrm{CN}$ $K=2$ emission in the same velocity intervals: $(88,90) \mathrm{km} \mathrm{s}^{-1}$ for the blue-shifted emission and $(103,107) \mathrm{km} \mathrm{s}^{-1}$ for the redshifted emission. As seen in the middle panel of Fig. 10, the $\mathrm{CH}_{3} \mathrm{CN} K=2$ high-velocity emission indeed traces the inner region of the molecular outflow, especially at blue-shifted velocities. We have also averaged the $\mathrm{CH}_{3} \mathrm{CN} v_{8}=1$ in the same high-velocity intervals, but the emission is very weak and mostly concentrated toward the center or the core, with no coincidence with the outflow lobes. This suggests that the velocity gradient traced in the lowest energy transitions is in part due to expansion (at least for the high velocities). On the other hand, the velocity gradient seen in the highest energy lines, coming from the innermost, hottest layers of the core, does not appear to be affected by the outflow and could be tracing rotation.

Following Cesaroni et al. (2011), we estimated the outflow parameters from the $\mathrm{SiO}$ and $\mathrm{CH}_{3} \mathrm{CN} v_{8}=1$ lines to check whether the velocity gradient traced in $\mathrm{CH}_{3} \mathrm{CN} v_{8}=1$ could be produced by the expansion of the outflow. In our calculations, we assumed a range of temperatures of $20-50 \mathrm{~K}$ for $\mathrm{SiO}$ and $100-250 \mathrm{~K}$ for vibrationally excited $\mathrm{CH}_{3} \mathrm{CN}$. The lower limit for the $\mathrm{SiO}$ temperature is set by the peak brightness temperature (see Fig. 5), while for $\mathrm{CH}_{3} \mathrm{CN} v_{8}=1$ the lower limit is the temperature adopted by Cesaroni et al. (2011) to calculate the parameters from $\mathrm{CH}_{3} \mathrm{CN} K=4$ and $\mathrm{CH}_{3}{ }^{13} \mathrm{CN} K=2$. The abundance of $\mathrm{CH}_{3} \mathrm{CN}$ relative to $\mathrm{H}_{2}$ was assumed to be equal to $10^{-8}$ (see e.g. van Dishoeck et al. 1993), while for $\mathrm{SiO}$ we used a range of $10^{-8}-10^{-7}$, following Codella et al. (2013). Table 3 gives the mass of the outflow, $M$, the momentum, $P$, the energy, $E$, and the corresponding rates obtained by dividing the previous quantities by the dynamical time scale of the outflow, $t_{\mathrm{dyn}} \simeq 6 \times 10^{3} \mathrm{yr}$, which has been calculated as $R_{\text {lobe }} / V_{\text {max }}$, where $R_{\text {lobe }}$ is the size $(\sim 0.15 \mathrm{pc})$ of the $\mathrm{SiO}$ lobes and $V_{\max }$ is the maximum velocity $\left(\sim 23 \mathrm{~km} \mathrm{~s}^{-1}\right)$ of the $\mathrm{SiO}$ outflow emission with respect to the systemic LSR velocity of $96.5 \mathrm{~km} \mathrm{~s}^{-1}$.

The outflow parameters estimated from $\mathrm{SiO}$ for a gas temperature of $50 \mathrm{~K}$ are similar to those calculated by Cesaroni et al. (2011) from ${ }^{12} \mathrm{CO}$ for a gas temperature of $100 \mathrm{~K}$, while those estimated from $\mathrm{CH}_{3} \mathrm{CN} v_{8}=1 K, l=(6,1)$ for a gas temperature 


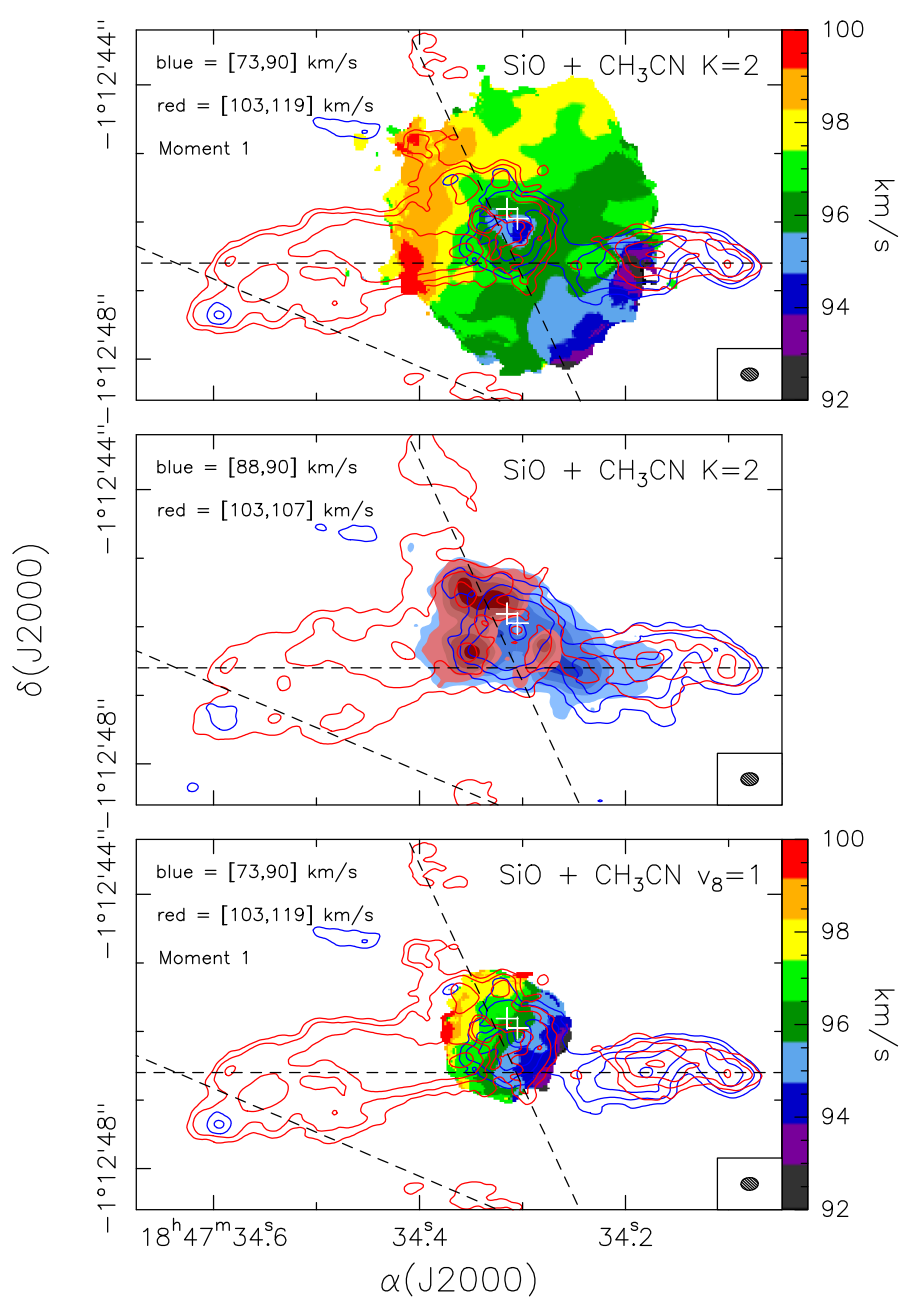

Fig. 10. Top panel: overlay of the line velocity (moment 1) map of $\mathrm{CH}_{3} \mathrm{CN} K=2$ (12-11) (colors) on the $\mathrm{SiO}(5-4)$ blue-shifted (blue contours) and red-shifted (red contours) averaged emission. The $\mathrm{CH}_{3} \mathrm{CN} K=2$ line velocity map has been computed over the velocity range $(92,100) \mathrm{km} \mathrm{s}^{-1}$. The velocity intervals over which the $\mathrm{SiO}$ emission has been averaged are indicated in the upper left-hand corner. Contour levels are the same as in Fig. 5. Middle panel: overlay of the $\mathrm{CH}_{3} \mathrm{CN} K=2(12-11)$ and $\mathrm{SiO}(5-4)$ blue-shifted and red-shifted emission averaged over the same velocity intervals, which are indicated in the upper left-hand corner. Contour and grayscale levels are 6, 18, 36 , and $72 \mathrm{mJy}$ beam $^{-1}$. Bottom panel: same as the top panel but for $\mathrm{CH}_{3} \mathrm{CN} K, l=(6,-1) v_{8}=1(12-11)$. The synthesized beam is shown in the lower right-hand corner. The white crosses indicate the positions of the two compact free-free continuum sources detected by Cesaroni et al. (2010). The black dashed lines indicate the direction of the three possible outflows.

of $250 \mathrm{~K}$ are similar to those obtained by Cesaroni et al. (2011) from $\mathrm{CH}_{3}{ }^{13} \mathrm{CN} K=2$ for a gas temperature of $100 \mathrm{~K}$. As seen in Table 3 , the outflow parameters estimated from vibrationally excited $\mathrm{CH}_{3} \mathrm{CN}$ are orders of magnitude higher than those estimated from $\mathrm{SiO}$, a typical outflow tracer, and therefore, not realistic. Such high values could only be consistent with those estimated, by means of single-dish observations, for pc-scale molecular outflows powered by O-type protostars (e.g., López-Sepulcre et al. 2009). In addition, we note that our ALMA observations can miss extended emission, and therefore, the values estimated by us from $\mathrm{CH}_{3} \mathrm{CN} v_{8}=1$ are likely lower limits.

Our results suggest that $\mathrm{CH}_{3} \mathrm{CN}$ and $\mathrm{SiO}$ are tracing different gas with different motions and confirm the results of Cesaroni
Table 4. Upper level energy and velocity of the red-shifted absorption with respect to the systemic LSR velocity.

\begin{tabular}{lcc}
\hline \hline \multicolumn{1}{c}{ Line } & $\begin{array}{c}E_{\text {up }} \\
(\mathrm{K})\end{array}$ & $\begin{array}{c}\left|V_{\mathrm{LSR}}-V_{\text {red }}\right| \\
\left(\mathrm{km} \mathrm{s}^{-1}\right)\end{array}$ \\
\hline $\mathrm{H}_{2} \mathrm{CO}\left(3_{0,3}-20,2\right)$ & 21 & $2.1 \pm 1.3$ \\
$\mathrm{H}_{2} \mathrm{CO}\left(3_{2,2}-2,1\right)$ & 68 & $4.0 \pm 1.3$ \\
$\mathrm{H}_{2} \mathrm{CO}\left(3_{2,1}-2,0\right)$ & 68 & $2.9 \pm 1.3$ \\
$\mathrm{CH}_{3} \mathrm{CN} K=2(12-11)$ & 97 & $5.1 \pm 0.2$ \\
$\mathrm{CH}_{3}{ }^{13} \mathrm{CN} K=2(12-11)$ & 97 & $5.8 \pm 0.2$ \\
${ }^{13} \mathrm{CH}_{3} \mathrm{CN} K=2(13-12)$ & 106 & $7.4 \pm 0.3$ \\
$\mathrm{CH}_{3} \mathrm{CN} K=3(12-11)$ & 133 & $5.1 \pm 0.2$ \\
${ }^{13} \mathrm{CH}_{3} \mathrm{CN} K=3(13-12)$ & 142 & $>7.6^{a}$ \\
$\mathrm{CH}_{3} \mathrm{CN} K=4(12-11)$ & 183 & $5.5 \pm 0.2$ \\
$\mathrm{CH}_{3}{ }^{13} \mathrm{CN} K=5(12-11)$ & 246 & $6.1 \pm 0.2$ \\
$\mathrm{CH}_{3} \mathrm{CN} K=5(12-11)$ & 247 & $>6.0^{a}$ \\
${ }^{13} \mathrm{CH}_{3} \mathrm{CN} K=5(13-12)$ & 256 & $8.4 \pm 0.3$ \\
$\mathrm{CH}_{3} \mathrm{CN} K=6(12-11)$ & 326 & $>5.7^{a}$ \\
$\mathrm{CH}_{3} \mathrm{CN} K=8(12-11)$ & 526 & $>6.0^{a}$ \\
$\mathrm{CH}_{3} \mathrm{CN} K, l=(3,-1) v_{8}=1(12-11)$ & 705 & $6.9 \pm 0.3$ \\
$\mathrm{CH}_{3} \mathrm{CN} K, l=(6,1) v_{8}=1(12-11)$ & 778 & $7.2 \pm 0.3$ \\
\hline
\end{tabular}

Notes. The velocity of the absorption feature has been measured toward the dust continuum peak and corresponds to that of the spectral channel with the lowest intensity at that position. ${ }^{(a)}$ Line slightly blended with other lines at frequencies lower than the rest frequency.

et al. (2011), who concluded that it is very unlikely that the velocity gradient traced in $\mathrm{CH}_{3} \mathrm{CN}$ and isotopologues is due to the expansion of the molecular gas. We conclude that this velocity gradient is produced by rotation of the core.

\subsection{Red-shifted absorption}

The most characteristic feature of the line emission toward the Main core in G31 is the inverse P-Cygni profile observed, although with different degrees of absorption, in basically all the species and at all energies. This red-shifted absorption was not detected in previous observations of the same lines, in particular $\mathrm{CH}_{3} \mathrm{CN}$ and $\mathrm{CH}_{3}{ }^{13} \mathrm{CN}$ (12-11) with the SMA and IRAM PdBI interferometers, due to the limited angular resolution. The detection of red-shifted absorption toward the core center (see Fig. 3) strongly supports the existence of infall in the core.

Following Beltrán et al. (2006), we estimated the infall rate inside a solid angle $\Omega$ for a radius of 1 '. $54\left(\sim 1.2 \times 10^{4} \mathrm{au}\right)$, which is that of the $\mathrm{CH}_{3} \mathrm{CN} K=2$ integrated emission (see top left panel of Fig. A.2), and an infall velocity, assumed to be equal to the difference between the velocity of the absorption feature and the systemic LSR velocity, of $5.1 \mathrm{~km} \mathrm{~s}^{-1}$. The choice of $\mathrm{CH}_{3} \mathrm{CN} \mathrm{K}=2$ is based on the fact that red-shifted absorption is very pronounced in this transition. The infall accretion rate is $\dot{M}_{\text {inf }}=\Omega /(4 \pi) 3 \times 10^{-2} M_{\odot} \mathrm{yr}^{-1}$. Such a high value is a factor 10 higher than that estimated from modeling (Osorio et al. 2009) for an infalling envelope of $2.3 \times 10^{4} \mathrm{au}$, but is consistent with the value estimated by Girart et al. (2009) at $345 \mathrm{GHz}$. Similar high infall rates have been estimated for other O-type (proto)stars (see Beltrán \& de Wit 2016).

\subsubsection{Accelerating infall}

To study whether the absorption changes with the energy of the line, we measured the velocity of the absorption feature in transitions spanning a broad range of energies at the position of the dust continuum peak. Table 4 gives the different species 


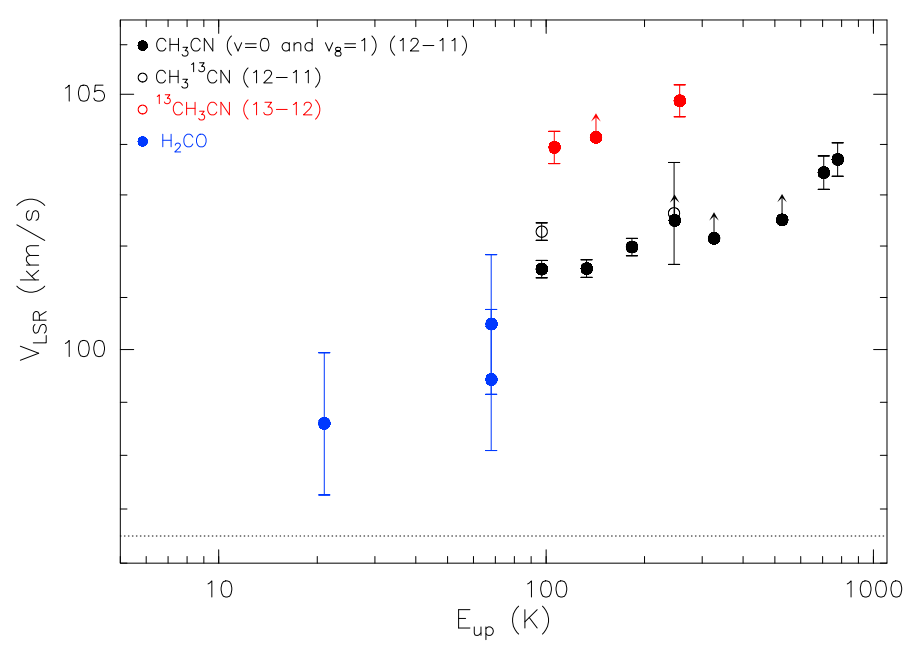

Fig. 11. Velocity of the absorption feature (measured toward the dust continuum peak) versus the upper level energy of the corresponding $\mathrm{CH}_{3} \mathrm{CN}, \mathrm{CH}_{3}{ }^{13} \mathrm{CN}$, and ${ }^{13} \mathrm{CH}_{3} \mathrm{CN}$, and $\mathrm{H}_{2} \mathrm{CO}$ transitions. The systemic LSR velocity is $96.5 \mathrm{~km} \mathrm{~s}^{-1}$ and is indicated with a dotted line. The black arrows indicate lower limits to the velocity and are for those lines that are slightly blended with other lines at frequencies lower than their rest frequency.

and transitions, the upper level energy of the line, and the difference between the velocity of the red-shifted absorption dip and the systemic LSR velocity $\left(96.5 \mathrm{~km} \mathrm{~s}^{-1}\right)$. The red-shifted velocity has only been calculated for those lines that are not heavily blended with other species, because the blending affects the estimate. For a few transitions that are slightly blended, we estimated a lower limit of the velocity. In Fig. 11, we plot the red-shifted velocity as a function of the upper level energy of the $\mathrm{CH}_{3} \mathrm{CN}$ (ground state and vibrationally excited) lines, $\mathrm{CH}_{3}{ }^{13} \mathrm{CN},{ }^{13} \mathrm{CH}_{3} \mathrm{CN}$, and $\mathrm{H}_{2} \mathrm{CO}$. The red-shifted velocity is lower for the $\mathrm{H}_{2} \mathrm{CO}$ transitions, which have the lowest energies. The $\mathrm{H}_{2} \mathrm{CO}$ lines are heavily affected by the absorption as suggested by both the extension and the depth of the absorption (see Fig. 4). In particular, $\mathrm{H}_{2} \mathrm{CO}\left(3_{0,3}-2_{0,2}\right)$, the lowest energy transition, shows no emission at the channel with the deepest absorption, and the brightness temperature $T_{\mathrm{B}}$ of the absorption is $-134 \mathrm{~K}$, comparable in absolute value to the brightness temperature of the continuum emission peak. Focusing on $\mathrm{CH}_{3} \mathrm{CN}$ and isotopologues, Table 4 and Fig. 11 show that the velocity of the absorption feature increases with the transition upper level energy, which suggests that the infall is accelerating toward the core center (where the temperature is higher), consistent with free-fall collapse. It should be noted that the red-shifted velocities of the ${ }^{13} \mathrm{CH}_{3} \mathrm{CN}(13-12)$ lines are slightly higher than those of $\mathrm{CH}_{3} \mathrm{CN}$ and $\mathrm{CH}_{3}{ }^{13} \mathrm{CN}(12-11)$ with same $K$ transition and similar energy. This suggests that the conditions to excite the (13-12) transitions occur deeper in the core and closer to the center. In any case, the increase of velocity with energy is found also if one considers only the (13-12) lines.

\subsubsection{Excitation conditions at the core center}

We used the maps with the continuum not subtracted to study the excitation conditions of the gas at the core center, namely, in the inner 0.22 of the core, where the absorption is strongest. As mentioned before, the species most affected by red-shifted absorption are $\mathrm{CH}_{3} \mathrm{CN}$ and isotopologues, and $\mathrm{H}_{2} \mathrm{CO}$. Because $\mathrm{CH}_{3} \mathrm{CN}$ has more transitions that can be fitted simultaneously than $\mathrm{H}_{2} \mathrm{CO}$, and the spectral resolution of the observations is higher $\left(0.33\right.$ to $0.66 \mathrm{~km} \mathrm{~s}^{-1}$ for methyl cyanide

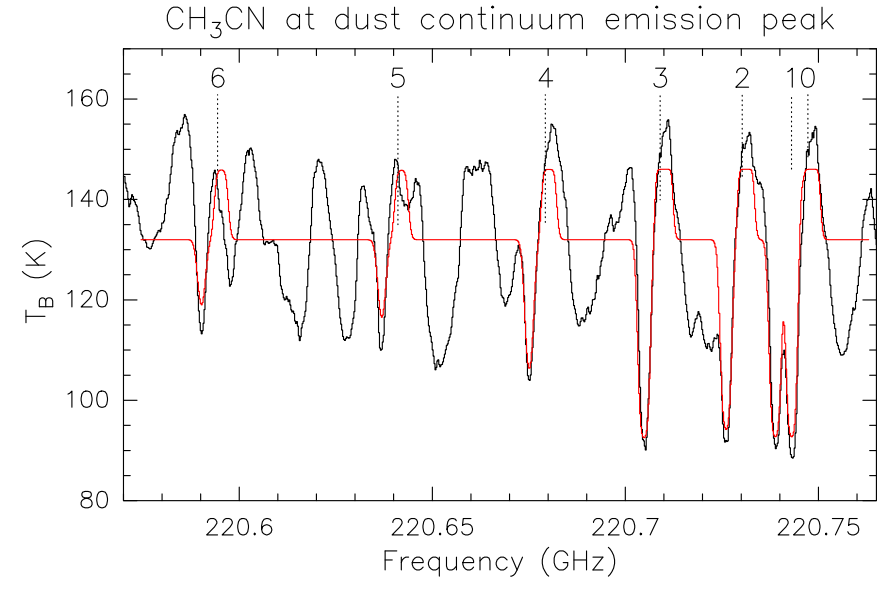

Fig. 12. Spectrum obtained at the dust continuum emission peak of the G31 Main core (black) and best fit to the $\mathrm{CH}_{3} \mathrm{CN}$ (12-11) $K=0$ to 6 transitions (red). The spectrum has been modeled with different components: i) a background layer with homogeneous intensity to describe the dust continuum core, ii) a core layer to describe the emission, and iii) a foreground layer located in front of the core component to describe the absorption (see Sect. 5.2.2). We note that the other emission and absorption lines not fitted correspond to other species.

and isotopologues, $2.7 \mathrm{~km} \mathrm{~s}^{-1}$ for formaldehyde), we modeled the absorption toward the center of the $\mathrm{G} 31$ Main core in $\mathrm{CH}_{3} \mathrm{CN}$ and its isotopologues. To estimate the physical parameters of the species, we modeled the spectrum at the central pixel with three different components: i) a background layer with uniform intensity to describe the dust continuum core, ii) a layer to describe the core emission, and iii) a foreground layer located in front of the core component to describe the absorption. For the modeling, we used XCLASS as explained in Sect. 4.1. The core and the foreground components are described with 5 parameters each: the source size $\theta$, the excitation temperature $T_{\text {ex }}$, the column density of the molecule, $N_{\text {tot }}$, the line width, $\Delta v$, and the offset velocity relative to the $V_{\mathrm{LSR}}, v_{\text {off }}$.

To fit the line emission of $\mathrm{CH}_{3} \mathrm{CN}$ and its isotopologues, the temperature of the continuum background layer was assumed to be constant and equal to the brightness temperature $T_{\mathrm{B}}=132 \mathrm{~K}$ measured at the dust continuum peak (see Table 1). We assumed that the foreground or absorption component is extended and that it fills the beam (i.e., no beam dilution is considered).

We started by fitting $\mathrm{CH}_{3} \mathrm{CN}$ and $\mathrm{CH}_{3} \mathrm{CN} v_{8}=1(12-11)$, and ${ }^{13} \mathrm{CH}_{3} \mathrm{CN}$ (13-12) simultaneously. XCLASS allows to simultaneously fit a species together with its isotopologues and vibrationally excited states by defining an isotopic ratio for each isotopologue. The transitions of $\mathrm{CH}_{3}{ }^{13} \mathrm{CN}$ (12-11) were not modeled because they are too blended with those of $\mathrm{CH}_{3} \mathrm{CN}$. Although we tried different approaches, like fixing the value of some parameters to reduce the number of free parameters, we could not find a satisfactory fit for all of them. This is probably due to the fact that the main species, the isotopologues, and the vibrationally excited states do not trace the same material and, thus, it is not possible to fit all of them with the same excitation conditions. Therefore, we decided to fit them separately.

The first to be fitted was $\mathrm{CH}_{3} \mathrm{CN}$, because it is more clearly affected by absorption (in particular the lower energy transitions). As shown in Fig. 2, $\mathrm{CH}_{3} \mathrm{CN}$ has been clearly detected up to the $K=8$ transition. For higher energy transitions ( $K=9$ and 10), the blending with other species is so severe that it is not possible to estimate their contribution. We fitted the spectra up to the $K=6$ component (see Fig. 12 and Table 5). The 
Table 5. Best fit physical parameters for the $\mathrm{CH}_{3} \mathrm{CN}(12-11) K=0$ to 6 transitions toward the center of the G31 core.

\begin{tabular}{cc}
\hline \hline Emission & Absorption \\
\hline$T_{e}=151_{+83}^{-8} \mathrm{~K}$ & $T_{\mathrm{a}}=97_{+3}^{-4} \mathrm{~K}$ \\
$\log N_{e}>17 \mathrm{~cm}^{-2}$ & $\log N_{\mathrm{a}}=15.9_{+0.3}^{-0.2} \mathrm{~cm}^{-2}$ \\
$\Delta v_{e}^{a}=3 \mathrm{~km} \mathrm{~s}^{-1}$ & $\Delta v_{\mathrm{a}}^{a}=3 \mathrm{~km} \mathrm{~s}^{-1}$ \\
$v_{\mathrm{off} e}^{a}=-1.3 \mathrm{~km} \mathrm{~s}^{-1}$ & $v_{\text {off a }}^{a}=5.5 \mathrm{~km} \mathrm{~s}^{-1}$ \\
\hline
\end{tabular}

Notes. ${ }^{(a)}$ Parameter fixed.

$K=7$ and 8 transitions could not be simultaneously fitted with the lower $K$ transitions, either because they are slightly blended with some lines of $\mathrm{CH}_{3}{ }^{13} \mathrm{CN}$ or because they could be tracing material with different excitation conditions than that traced by the lower energy transitions. To reduce the number of free parameters, we fixed the line width $(\Delta v)$ and the offset velocity relative to the $V_{\mathrm{LSR}}\left(v_{\mathrm{off}}\right)$ for both emission and absorption. We note that the fact that XCLASS assumes that $v_{\text {off }}$ is the same for all the transitions is questionable, because as we just discussed, the velocity of the absorption feature increases with the energy of the transition (Table 4 and Fig. 11). As for $\Delta v$, it should also change with the energy of the transition, but in this case, the change is less evident and, therefore, the approximation of constant $\Delta v$ is reasonable. We assumed that the source is extended and fills the beam size, like for the foreground component (the value obtained if the size parameter is left free is much larger than the beam size). The best fit model indicates that the temperature of the emission layer $(151 \mathrm{~K})$ is higher than that of the dust emission $(132 \mathrm{~K})$, while that of the absorption layer is lower $(97 \mathrm{~K})$. As shown in Fig. 12, the $\mathrm{CH}_{3} \mathrm{CN}$ modeled in emission is slightly saturated, as suggested by the fact that the $K=0,1$, 2 , and 3 transitions have almost the same brightness temperature despite their different energies. Therefore, the best fit column density of the emission component, $N_{e}=1 \times 10^{17} \mathrm{~cm}^{-2}$, should be taken as a lower limit. This column density is one order of magnitude higher than that of the absorbing layer.

The isotopologue ${ }^{13} \mathrm{CH}_{3} \mathrm{CN}$ (13-12) has been clearly detected up to the $K=6$ transition. We fitted the spectra up to this transition avoiding the $K=5$ transition, which is highly blended with other species (see Fig. 13). The source was assumed to be extended in both emission and absorption (i.e., no correction for beam dilution is applied). The fit to the absorption was highly dependent on the initial guesses and it is not as good as that to the absorption in $\mathrm{CH}_{3} \mathrm{CN}$ (see Figs. 12 and 13). Therefore, in Table 6 we only give the best fit parameters of the emission, which have been obtained by fixing the line width and the velocity offset. The excitation temperature of the emission is similar, within the uncertainties, to that obtained for $\mathrm{CH}_{3} \mathrm{CN}$. Regarding the column density of ${ }^{13} \mathrm{CH}_{3} \mathrm{CN}$, the value obtained is only about two times lower than that of $\mathrm{CH}_{3} \mathrm{CN}$. This indicates a very low $\left[\mathrm{CH}_{3} \mathrm{CN}\right] /\left[{ }^{13} \mathrm{CH}_{3} \mathrm{CN}\right]$ relative abundance, especially if one takes into account that the relative abundance of $\left[{ }^{12} \mathrm{C} /{ }^{13} \mathrm{C}\right]$ at the galactocentric distance of $4.5 \mathrm{kpc}$ of G31 would be 47 (Milam et al. 2005). We note that the column density of $\mathrm{CH}_{3} \mathrm{CN}$ in emission should be taken as a lower limit because the emission is saturated. This could explain in part the low values obtained in emission for the $\left[{ }^{12} \mathrm{C} /{ }^{13} \mathrm{C}\right]$ ratio, although the ${ }^{13} \mathrm{CH}_{3} \mathrm{CN}$ emission also seems slightly saturated (see Fig. 13).

We cannot disregard the possibility that the $\left[{ }^{12} \mathrm{C} /{ }^{13} \mathrm{C}\right]$ ratio in the G31 core is unusually low. To investigate this possibility we fitted both the $\mathrm{CH}_{3} \mathrm{CN}$ and ${ }^{13} \mathrm{CH}_{3} \mathrm{CN}$ emission in a ring located at a distance of 1 '. 32 from the center of the core, where

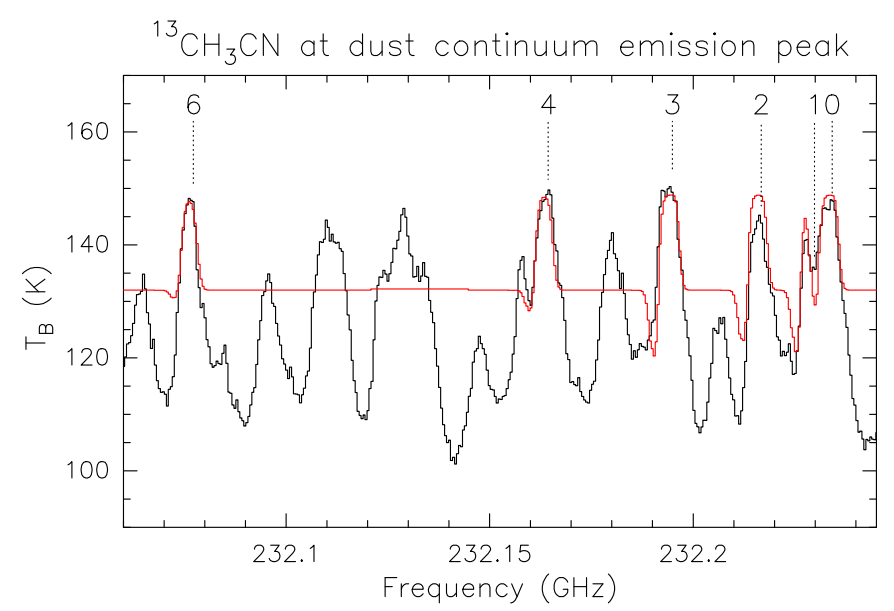

Fig. 13. Same as Fig. 12 for ${ }^{13} \mathrm{CH}_{3} \mathrm{CN}(12-11) K=0$ to 6 transitions.

Table 6. Best fit physical parameters for the ${ }^{13} \mathrm{CH}_{3} \mathrm{CN}(12-11) \mathrm{K}=0$ to 6 transitions toward the center of the G31 core.

\begin{tabular}{c}
\hline Emission \\
\hline$T_{e}=155_{+107}^{-50} \mathrm{~K}$ \\
$\log N_{e}=16.8_{+0.1}^{-1.2} \mathrm{~cm}^{-2}$ \\
$\Delta v_{e}^{a}=3.5 \mathrm{~km} \mathrm{~s}^{-1}$ \\
$v_{\mathrm{off} e}^{a}=1.3 \mathrm{~km} \mathrm{~s}^{-1}$ \\
\hline
\end{tabular}

Notes. ${ }^{(a)}$ Parameter fixed.

the absorption is negligible. For this ring, the temperature of the background layer was assumed to be $1.8 \mathrm{~K}$, which is the continuum brightness temperature at that radius. The best fit indicates a similar excitation temperature, within the errors, for both $\mathrm{CH}_{3} \mathrm{CN}$ and ${ }^{13} \mathrm{CH}_{3} \mathrm{CN}$, but a column density a factor ten lower $\left(\log N_{e}=14.3_{+1.7}^{-0.2} \mathrm{~cm}^{-2}\right)$ for ${ }^{13} \mathrm{CH}_{3} \mathrm{CN}$ than for $\mathrm{CH}_{3} \mathrm{CN}$ $\left(\log N_{e}=15.3_{+1.3}^{-0.01} \mathrm{~cm}^{-2}\right) . \mathrm{A}\left[{ }^{12} \mathrm{C} /{ }^{13} \mathrm{C}\right]$ ratio of ten is greater than the value obtained toward the central position but still significantly less than the typical value. To check wether this low ratio is also seen in other species, we estimated the column densities of $\mathrm{CH}_{3} \mathrm{OCHO}$ and $\mathrm{CH}_{3} \mathrm{O}^{13} \mathrm{CHO}$ in the rings not affected by absorption and obtained $\left[{ }^{12} \mathrm{C} /{ }^{13} \mathrm{C}\right] \simeq 10$. This indicates that in the $\mathrm{G} 31$ core there is an overabundance of ${ }^{13} \mathrm{C}$ with respect to ${ }^{12} \mathrm{C}$.

The $\mathrm{CH}_{3} \mathrm{CN} v_{8}=1(12-11)$ transitions at the central position have been fitted only with an emission component because an additional foreground absorbing layer produces a negligible change in the fit. This is consistent with the fact that the red-shifted absorption is less evident for these high energy transitions. The best fit parameters are given in Table 7. The excitation temperature of the vibrationally excited transitions is consistent with that obtained for $\mathrm{CH}_{3} \mathrm{CN}$ and ${ }^{13} \mathrm{CH}_{3} \mathrm{CN}$, but the column density is almost one order of magnitude higher than that of the ground state. Therefore, we decided to fit the $\mathrm{CH}_{3} \mathrm{CN} v_{8}=1$ emission by fixing $N_{e}$ to a value of $1 \times 10^{17} \mathrm{~cm}^{-2}$, obtained for the ground state $\mathrm{CH}_{3} \mathrm{CN}$, and got $T_{e}=224 \mathrm{~K}$. Despite the large uncertainties in the fit, the fact that one needs either a much higher column density or a much higher excitation temperature to model the vibrationally excited transitions suggests that these high energy transitions are likely tracing hotter and denser material located deeper in the core.

Because of the existence of temperature and density gradients in the G31 core, as discussed in Sect. 4.2, the $\mathrm{CH}_{3} \mathrm{CN}$ 
Table 7. Best fit physical parameters for the $\mathrm{CH}_{3} \mathrm{CN} v_{8}=1$ (12-11) transitions toward the center of the G31 core.

\begin{tabular}{cc}
\hline \hline Emission & $\begin{array}{c}\text { Emission } \\
\text { (fixing } N_{e} \text { ) }\end{array}$ \\
\hline$T_{e}=165_{+64}^{-153} \mathrm{~K}$ & $T_{e}=224_{+3}^{-208} \mathrm{~K}$ \\
$\log N_{e}=17.7_{+1.24}^{-2.9} \mathrm{~cm}^{-2}$ & $\log N_{e}^{a}=17 \mathrm{~cm}^{-2}$ \\
$\Delta v_{e}^{a}=6 \mathrm{~km} \mathrm{~s}^{-1}$ & $\Delta v_{e}^{a}=6 \mathrm{~km} \mathrm{~s}^{-1}$ \\
$v_{\mathrm{off} e}=0.5_{+5.2}^{-2.7} \mathrm{~km} \mathrm{~s}^{-1}$ & $v_{\mathrm{off} e}=0.6_{+0.15}^{-2.3} \mathrm{~km} \mathrm{~s}^{-1}$ \\
\hline
\end{tabular}

Notes. ${ }^{(a)}$ Parameter fixed.

$v_{8}=1$ emission cannot be well represented by the same excitation conditions as those of the ground state emission. Radiative pumping by IR photons may also play a role in the excitation of $\mathrm{CH}_{3} \mathrm{CN} v_{8}=1$ (e.g., Goldsmith et al. 1983). To carry out an analysis of collisional excitation and check whether collisional processes could produce the observed excitation of the $v_{8}$ transitions it is necessary to know the spontaneous decay rate, $A_{\text {vib }}$, and the collision cross sections for vibrational excitation $\langle\sigma v\rangle$ vib. Using the estimates of $A_{\mathrm{vib}}=1.6 \times 10^{-2} \mathrm{~s}^{-1}$ of Koivusaari et al. (1992), and assuming the same collisional excitation rate as for the vibrational ground state, $\langle\sigma v\rangle$ vib $=$ $2 \times 10^{-10} \mathrm{~cm}^{3} \mathrm{~s}^{-1}$ (Green et al. 1986), then collisional excitation is possible only for $n \gtrsim 10^{8} \mathrm{~cm}^{-3}$. Because $n$ estimated in the core ranges from $5.4 \times 10^{6} \mathrm{~cm}^{-3}$ for $R=1^{\prime \prime}$. 076 to $\simeq 1 \times 10^{8} \mathrm{~cm}^{-3}$ for $R=0.22$, we conclude that the vibrational levels could be radiatively pumped. The first vibrationally excited state $v_{8}=1$ is at $525 \mathrm{~K}$ above the $\mathrm{CH}_{3} \mathrm{CN}$ ground vibrational state and can be pumped by $27 \mu \mathrm{m}$ photons.

\subsection{A monolithic core?}

The most striking characteristic of the G31 Main core is its compact appearance. As seen in Fig. 1, the dust continuum emission does not show any hint of fragmentation although the core is well resolved in our maps. The presence of red-shifted absorption in the core would suggest that what we are observing in G31 is a "real" massive core undergoing monolithic collapse, as suggested by McKee et al. (2002). However, one cannot discard the possibility that this homogeneous and monolithic appearance is due to a combination of large opacity of the $217 \mathrm{GHz}$ continuum emission and insufficient angular resolution. One may argue that the size of a typical fragment should be of the same order as the separation between the two free-free sources detected inside the core, which is $\sim 0.2$ or $\sim 1600 \mathrm{au}$. By using Eqs. (1) and (5), we have estimated the temperature and volume density in the inner part of the core $(R \leq 0.22)$, and the thermal Jeans length, and found that it ranges from $\sim 1300$ to $2000 \mathrm{au}$. Therefore, it is comparable to the separation of $\sim 1600$ au of the free-free sources. This implies that fragmentation, if present as expected from theoretical calculations (see Peters et al. 2010b), cannot be properly imaged with the current observations.

One must also take into account that the continuum emission observed by us at $217 \mathrm{GHz}$ is optically thick (the brightness temperature of the continuum peak, $\sim 132 \mathrm{~K}$, is comparable to the dust temperature, $\gtrsim 150 \mathrm{~K}$ ). The high opacity of G31 has already been determined by Rivilla et al. (2017) based on multiwavelength observations. Such a large opacity $(\lesssim 2)$ is likely to mask any inhomogeneity of the core. This result is similar to what is found in the most massive core of $\operatorname{SgrB2}(\mathrm{N})$. Sub-arcsecond resolution observations with the SMA $(345 \mathrm{GHz}$; Qin et al. 2011) and with ALMA (250 GHz; Sánchez-Monge et al. 2017) revealed a core with monolithic appearance and a radius of about $6000 \mathrm{au}$. In this case, 3D radiative transfer modeling of the region (Schmiedeke et al. 2016) suggests that the emission at frequencies above $200 \mathrm{GHz}$ is optically thick when observed at an angular resolution $<0.5^{\prime \prime}$.

These results altogether pose questions about the nature of the G31 Main core. On the one hand, Girart et al. (2009) have revealed the presence of a magnetic field uniformly threading the core, with a strength in the plane of the sky of $\sim 10 \mathrm{mG}$. This, together with the linear increase of rotation velocity with radius (suggested by each PV plot in Fig. 8), and the (apparent) homogeneity of the core, point to a monolithic core stabilized by the magnetic field and undergoing solid-body rotation. On the other hand, a mass-to-magnetic flux ratio of $\sim 2.7$ times the critical value for collapse (Girart et al. 2009), the presence of redshifted absorption, the existence of two embedded massive stars (the two free-free sources), and the rotational spin-up toward the core center (suggested by Fig. 9) are consistent with an unstable core undergoing fragmentation with infall and differential rotation due to conservation of angular momentum. We believe that an optically thick core with unresolved clumpiness and undergoing infall and rotation spin-up can explain all these features, as previously discussed.

Future approved ALMA observations at a much higher angular resolution of $\sim 0$.' $^{\prime} 08$, corresponding to $\sim 600$ au at the distance of the source, will help us to reveal the existence of clumpiness and thus confirm whether G31 is indeed a dynamically collapsing core undergoing global fragmentation.

\section{Conclusions}

As part of our continuing effort to search for circumstellar disks around high-mass young stellar objects, we have observed the O-type star-forming region G31.41+0.31 with ALMA at $1.4 \mathrm{~mm}$. The angular resolution $(\sim 0.2)$ of the observations has allowed us to study the G31 HMC in unprecedented detail, potentially tracing structures as small as $\sim 1600$ au.

The dust continuum emission has been resolved into two cores, a Main core chemically rich that peaks close to the position of the two unresolved free-free continuum sources detected by Cesaroni et al. (2010), and a much weaker and smaller NE core. The dust continuum emission of the Main core looks featureless in our observations, although it has an average halfpower diameter of $\sim 5300 \mathrm{au}$, much greater than our resolution $(\sim 1600 \mathrm{au})$.

The $\mathrm{SiO}$ emission reveals the presence of at least three outflows associated with the G31 core: an E-W outflow to the south of the dust continuum emission peak of the Main core and not associated with any of the two embedded free-free sources; a N-S outflow associated with water masers, which could be driven by the millimeter source associated with the dust continuum emission peak of the Main core or by one of the two free-free sources embedded in this core; and a NE-SW outflow to the south of the Main core and not associated with it.

The high sensitivity of the ALMA observations has allowed us to detect several transitions of the high-density tracer $\mathrm{CH}_{3} \mathrm{OCHO}$, both ground state and torsionally excited, and this has allowed us to estimate the physical parameters of the Main core. The LTE modeling of the $\mathrm{CH}_{3} \mathrm{OCHO}$ emission indicates that there is an excitation temperature gradient in the core, with $T_{\text {ex }} \propto R^{-0.77}$, consistent with the profiles of hot molecular cores modeled by Nomura \& Millar (2004) and Osorio et al. (2009). The $\mathrm{CH}_{3} \mathrm{OCHO}$ column density $\left(\mathrm{N}_{\mathrm{CH}_{3} \mathrm{OCHO}}\right)$ profile in the core is consistent with a broken power law, with the inner part of 
the core having $N_{\mathrm{CH}_{3} \mathrm{OCHO}} \propto R^{-0.82}$, and the outer part being considerably steeper, with $N_{\mathrm{CH}_{3} \mathrm{OCHO}} \propto R^{-4.63}$. On the other hand, the $\mathrm{H}_{2}$ column density profile, estimated from the dust continuum emission, does not show any sharp drop in the outer part of the core, with $N_{\mathrm{H}_{2}} \propto R^{-1.15}$. We conclude that the drop in $\mathrm{CH}_{3} \mathrm{OCHO}$ abundance is real. This suggests that $\mathrm{CH}_{3} \mathrm{OCHO}$ is concentrated in the inner regions $(R<5000 \mathrm{au})$ of the G31 Main core, and confirms that complex organic molecules are reliable tracers of the gas located close to the forming protostars. The mass of the G31 Main core, estimated assuming that the dust emission is in the Rayleigh-Jeans regime and that the density and temperature of the core follow the previously established powerlaw distributions, is $\sim 120 M_{\odot}$, while the mean volume density at the radius of the core $(8500 \mathrm{au})$ is $\sim 5.4 \times 10^{6} \mathrm{~cm}^{-3}$.

The emission of $\mathrm{CH}_{3} \mathrm{CN}$ and $\mathrm{CH}_{3} \mathrm{OCHO}$, both ground state and vibrationally excited, and of $\mathrm{CH}_{3} \mathrm{CN}$ isotopologues in the Main core shows a clear velocity gradient along the NE-SW direction consistent with what was previously found by Beltrán et al. (2004) and Cesaroni et al. (2011). The velocity appears to increase linearly with distance from the core center, consistent with solid-body rotation. However, the PV plots along the major axis of the core indicate that the velocities increase for increasing energies. This suggests that the rotation spins up toward the center, as expected for conservation of angular momentum in a rotating and infalling structure, which appears initially inconsistent with the solid-body rotation. A possible explanation for this could be that each line is tracing only a relatively narrow annulus of material. The projection of the rotation velocity along the line of sight increases linearly with distance from the star, mimicking solid-body rotation, while at the same time, higher energy lines trace smaller annuli, closer to the star and thus rotating faster.

The line emission toward the dust continuum peak of the Main core shows inverse P-Cygni profiles in basically all the species and at all energies, although the $\mathrm{H}_{2} \mathrm{CO}$ and lowest-energy $\mathrm{CH}_{3} \mathrm{CN}$ transitions show the strongest absorption. The detection of red-shifted absorption toward the core center supports the existence of infall in the core. The velocity of the absorption feature (which is a good proxy for the infall velocity) becomes progressively more red shifted with increasing energy of the transition. This suggests that the infall is accelerating toward the center of the core, consistent with free-fall collapse. The excitation conditions of the emitting and absorbing gas components at the position of the dust continuum emission peak have been estimated by modeling the $\mathrm{CH}_{3} \mathrm{CN}$ and ${ }^{13} \mathrm{CH}_{3} \mathrm{CN}$ transitions, assuming LTE. We find that the column density of the absorbing layer is more than one order of magnitude smaller than that of the emitting layer. We also find that the $\left[{ }^{12} \mathrm{C} /{ }^{13} \mathrm{C}\right]$ ratio in the G31 core is unusually low, suggesting that there is an overabundance of ${ }^{13} \mathrm{C}$ with respect to ${ }^{12} \mathrm{C}$. The modeling of the $\mathrm{CH}_{3} \mathrm{CN}$ $v_{8}=1$ emission at the central position indicates that to excite these transitions either a much higher column density or a much higher excitation temperature than that needed to populate the ground state levels of $\mathrm{CH}_{3} \mathrm{CN}$ is necessary. This suggests that $\mathrm{CH}_{3} \mathrm{CN} v_{8}=1$ could be excited via radiative pumping by mid-IR $(27 \mu \mathrm{m})$ photons.

Although its homogeneous appearance suggests that G31 Main could be a true monolithic core, as predicted by McKee et al. (2002), the presence of red-shifted absorption, the existence of two embedded massive stars at the center, and the rotational spin-up are consistent with an unstable core undergoing fragmentation with infall and differential rotation due to conservation of angular momentum. Therefore, we argue that the most likely explanation for its morphology is that the dust emission is optically thick, as suggested by the detection of red-shifted absorption, and this prevents the detection of any inhomogeneity in the core.

Acknowledgements. We thank the referee, Dr. Paul Goldsmith, for the careful reading of the manuscript. This paper makes use of the following ALMA data: ADS/JAO.ALMA\#2013.1.00489.S. ALMA is a partnership of ESO (representing its member states), NSF (USA), and NINS (Japan), together with NRC (Canada), and NSC and ASIAA (Taiwan), in cooperation with the Republic of Chile. The Joint ALMA Observatory is operated by ESO, AUI/NRAO, and NAOJ. This work was partly supported by the Italian Ministero dell'Istruzione, Università e Ricerca through the grant Progetti Premiali 2012 iALMA (CUP C52I13000140001), by the Deutsche Forschungs-Gemeinschaft (DFG, German Research Foundation) - Ref no. FOR 2634/1 TE 1024/1-1, and by the DFG cluster of excellence Origin and Structure of the Universe (http://www.universe-cluster.de). V.M.R. has received funding from the European Union's Horizon 2020 research and innovation program under the Marie Skłodowska-Curie grant agreement No 664931. Á.S.M. and P.S. are partially supported by the Deutsche Forschungsgemeinschaft through grant SFB 956 (subproject A6). XCLASS development is supported by BMBF/Verbundforschung through the Projects ALMA-ARC 05A11PK3 and 05A14PK1 and through ESO Project 56787/14/60579/HNE. A.A., H.B., and J.C.M. acknowledge support from the European Research Council under the European Community's Horizon 2020 framework program (2014-2020) via the ERC Consolidator grant "From Cloud to Star Formation (CSF)" (project number 648505). R.G.M. acknowledges support from UNAM-PAPIIT program IA102817. A.K. and R.K. acknowledge financial support via the Emmy Noether Research Programme funded by the German Research Foundation (DFG) under grant no. KU 2849/3-1. M.S.N.K. acknowledges the support from Fundação para a Ciência e Tecnologia (FCT) through Investigador FCT contracts IF/00956/2015/CP1273/CT0002, and the H2020 Marie-Curie Intra-European Fellowship project GESTATE (661249).

\section{References}

Adams F. C., \& Shu F. H. 1986, ApJ, 308, 836

Araya, E., Hofner, P., Kurtz, S., Olmi, L., \& Linz, H. 2008, ApJ, 675, 420

Beltrán, M. T., \& de Wit, W. J. 2016, A\&ARv, 24, 6

Beltrán, M. T., Estalella, R., Ho, P. T. P., et al. 2002, ApJ, 565, 1069

Beltrán, M. T., Cesaroni, R., Neri, R., et al. 2004, ApJ, 601, L190

Beltrán, M. T., Cesaroni, R., Neri, R., et al. 2005, A\&A, 435, 901

Beltrán, M. T., Cesaroni, R., Codella, C., et al. 2006, Nature, 443, 427

Beltrán, M. T., Codella, C., Viti, S., Neri, R., \& Cesaroni, R. 2009, ApJ, 690, L93

Beltrán, M. T., Sánchez-Monge, Á., Cesaroni, R., et al. 2014, A\&A, 571, A52

Bonnell, I. A., \& Bate, M. R. 2006, MNRAS, 370, 488

Briggs, D. 1995, Ph.D. Thesis, New Mexico Inst. of Mining Tech., USA

Burke, D. J., Puletti, F., Brown, W. A., et al. 2015, MNRAS, 447, 1444

Cesaroni, R., Olmi, L., Walmsley, C. M., Churchwell, E., \& Hofner, P. 1994, ApJ, 435, L137

Cesaroni, R., Hofner, P., Araya, E., \& Kurtz, S. 2010, A\&A, 590, A50

Cesaroni, R., Beltrán, M. T., Zhang, Q., Beuther, H., \& Fallscheer, C. 2011, A\&A, 533, A73

Cesaroni, R., Sánchez-Monge, Á., Beltrán, M. T., et al. 2017, A\&A, 602, A59

Churchwell, E., Walmsley, C. M., \& Cesaroni, R. 1990, A\&AS, 83, 119

Codella, C., Beltrán, M. T., Cesaroni, R., et al. 2013, A\&A, 550, A81

Gibb, A. G., Wyrowski, F., \& Mundy, L. G. 2004, ApJ, 616, 301

Girart, J. M., Beltrán, M. T., Zhang, Q., Rao, R., \& Estalella, R. 2009, Science, 324, 1408

Goldsmith, P. F., Krotkov, R., Snell, R. N., Brown, R. D., \& Godfrey, P. 1983, ApJ, 274, 184

Green, S. 1986, ApJ, 309, 331

Ilee, J. D., Cyganowski, C. J., Nazari, P., et al. 2016, MNRAS, 462, 4386

Jijina, J., \& Adams, F. C. 1996, ApJ, 462, 874

Johnston, K. G., Robitaille, T. P., Beuther, H., et al. 2015, ApJ, 813, L19

Kahn, F. 1974, A\&A, 37, 149

Klassen, M., Pudritz, R. E., Kuiper, R., Peters, Th., \& Banerjee, R. 2016, ApJ, 823,28

Krumholz, M. R., Klein, R. I., McKee, C. F., Offner, S. S. R., \& Cunningham, A. J. 2009, Science, 323, 754

Koivusaari, M., Horneman, V.-M., \& Anttila, R. 1992, J. Mol. Spectr 152, 377

Kuiper R., \& Yorke, H. W. 2013, ApJ, 763, 104

Kuiper R., Klahr, H., Beuther, H., \& Henning, T. 2010, ApJ, 722, 1556

Kuiper R., Klahr, H., Beuther, H., \& Henning, T. 2011, ApJ, 732, 20

López-Sepulcre, A., Codella, C., Cesaroni, R., Marcelino, N., \& Walmsley, C. M. 2009, A\&A, 499, 811

Mayen-Gijon, J. M., Anglada, G., Osorio, M., et al. 2014, MNRAS, 437, 3766

McKee, C. F., \& Tan, J. C. 2002, Nature, 416, 59 
A\&A 615, A141 (2018)

McMullin, J. P., Waters, B., Schiebel, D., Young, W., \& Golap, K. 2007, ASP Conf. Ser., 376, 127

Milam, S. N., Savage, C., Brewster, M. A., \& Ziurys, L. M. 2005, ApJ, 634, 1126

Möller, T., Endres, C., \& Schilke, P. 2017, A\&A, 598, A7

Moscadelli, L., Li, J. J., Cesaroni, R., et al. 2013, A\&A, 549, A122

Nakano, T. 1989, ApJ, 345, 464

Nomura, H., \& Millar, T. J. 2004, A\&A, 414, 409

Nguyen-Luong, Q., Mote, F., Schuller, F., et al. 2011, A\&A, 529, A41

Olmi, L., Cesaroni, R., \& Walmsley, C. M. 1996, A\&A, 307, 599

Osorio, M., Anglada, G., Lizano, S., \& D’Alessio, P. 2009, ApJ, 694, 29

Ossenkopf, V., \& Henning, Th. 1994, A\&A, 291, 943

Peters, Th., Banerjee, R., Klessen, R. S., et al. 2010a, ApJ, 711, 1017

Peters, Th., Klessen, R. S., Mac Low M.-M., \& Banerjee, R. 2010b, ApJ, 725,134

Qin, S.-L., Schilke, P., Rolffs, R., et al. 2011, A\&A, 530, L9

Reid, M. J., Menten, K. M., Brunthaler, A., et al. 2014, ApJ, 783, 130
Rivilla, V. M., Fontani, F., Beltrán, M. T., et al. 2016, ApJ, 826, 161 Rivilla, V. M., Beltrán, M. T., Cesaroni, R., et al. 2017, A\&A, 598, A59 Sánchez-Monge, Á., Schilke, P., Schmiedeke, A., et al. 2017, A\&A, 604, A6 Sánchez-Monge, Á., Schilke, P., Ginsburg, A., Cesaroni, R., \& Schmiedeke, A. 2018, A\&A, 609, A101

Schmiedeke, A., Schilke, P., Möller, Th., et al. 2016, A\&A, 588, A143

Shakura, N. I., \& Sunyaev, R. A. 1973, A\&A, 24, 337

van der Tak, F. S., van Dishoeck, E. F., Evans, N. J., \& Balke, G. 2000, ApJ, 537, 303

van Dishoeck, E. F., Blake, G. A., Draine, B. T., \& Lunine, J. I. 1993, Protostars and Planets III, eds. E. H. Levy, \& J. I. Lunine, (Tucson: Univ. of Arizona Press), 163

Wolfire, M, \& Cassinelli, J. 1987, ApJ, 319, 850

Wyrowski, F., Güsten, R., Menten, K. M., et al. 2012, A\&A, 542, L15

Yorke, H. W., \& Krügel, E. 1977, A\&A, 54, 183

Zhang, B., Moscadelli, L., Sato, M., et al. 2014, ApJ, 781, 89 


\section{Appendix A:}
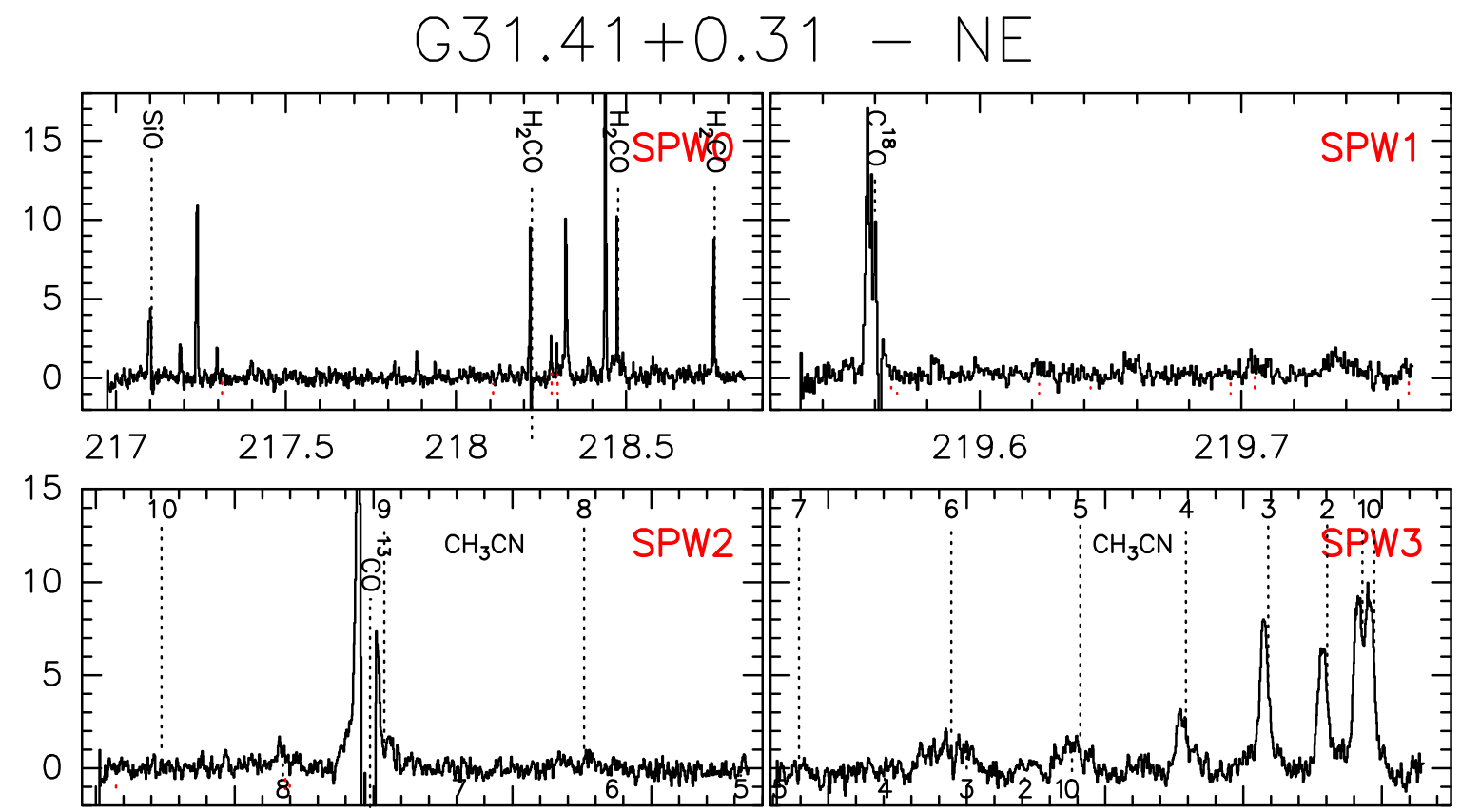

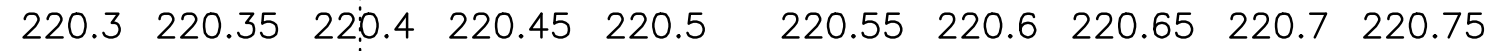
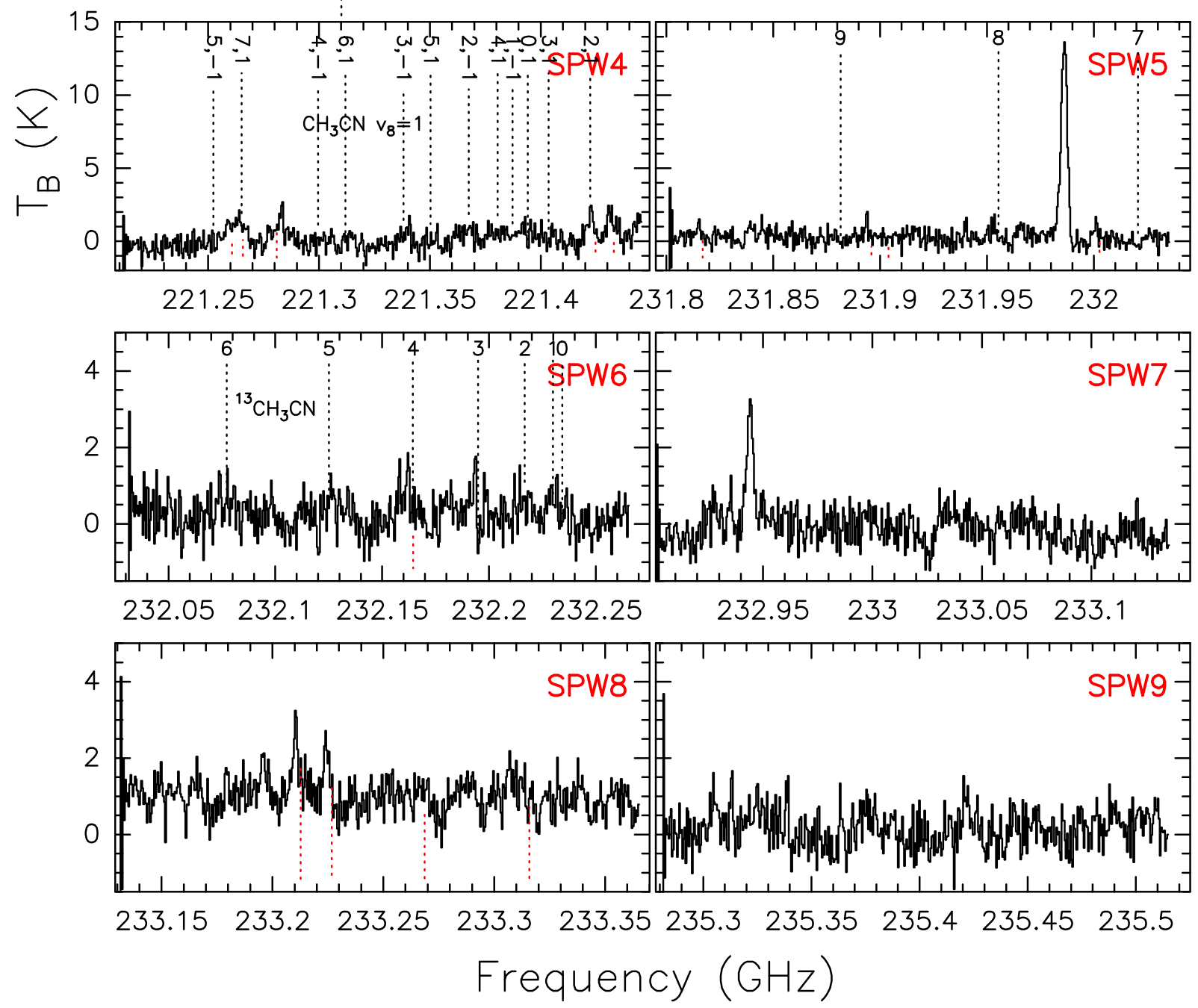

Fig. A.1. Same as Fig. 2 for the NE source. 
A\&A 615, A141 (2018)

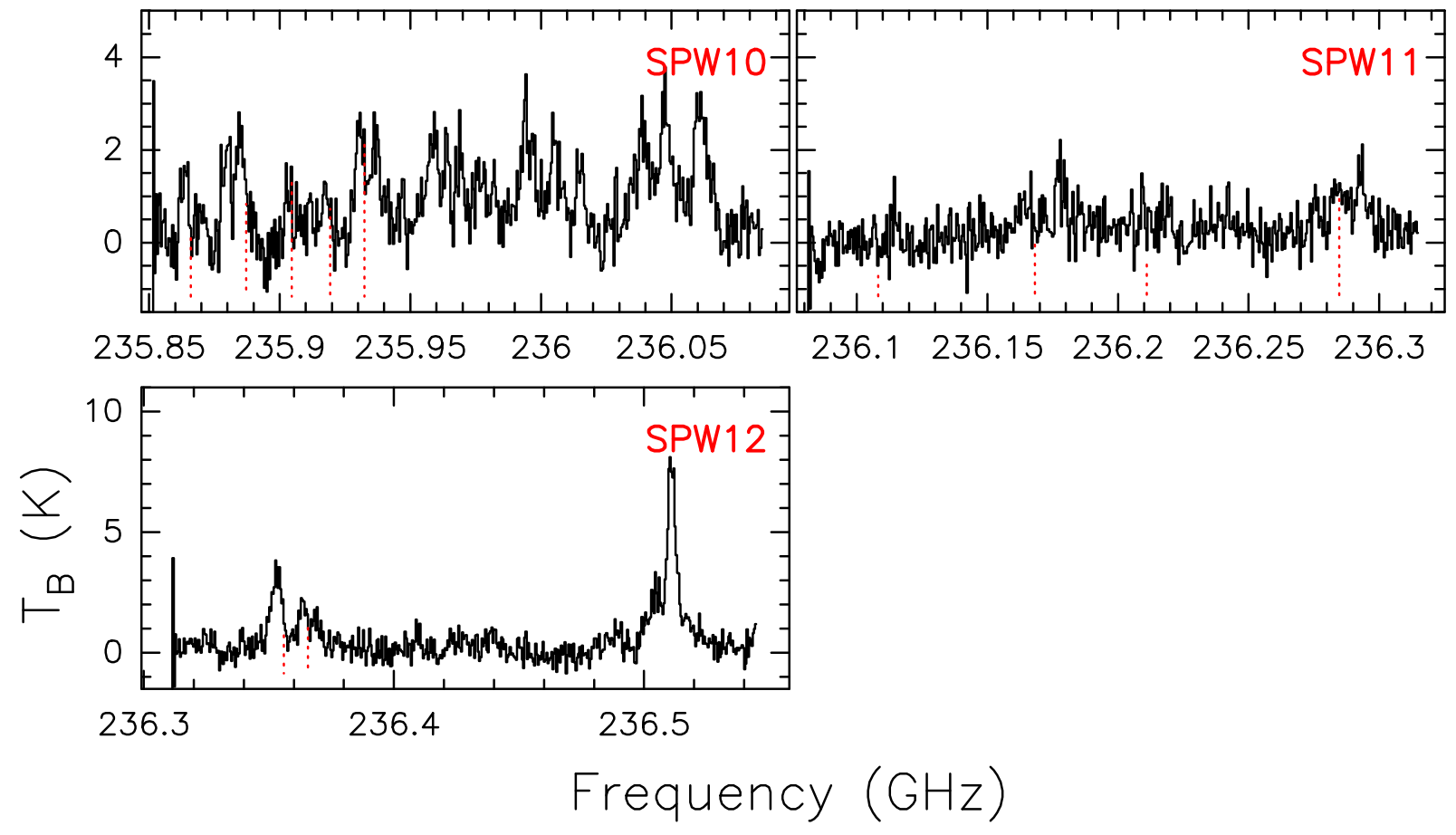

Fig. A.1. continued 
M. T. Beltrán et al.: Infall and rotation in G31.41+0.31

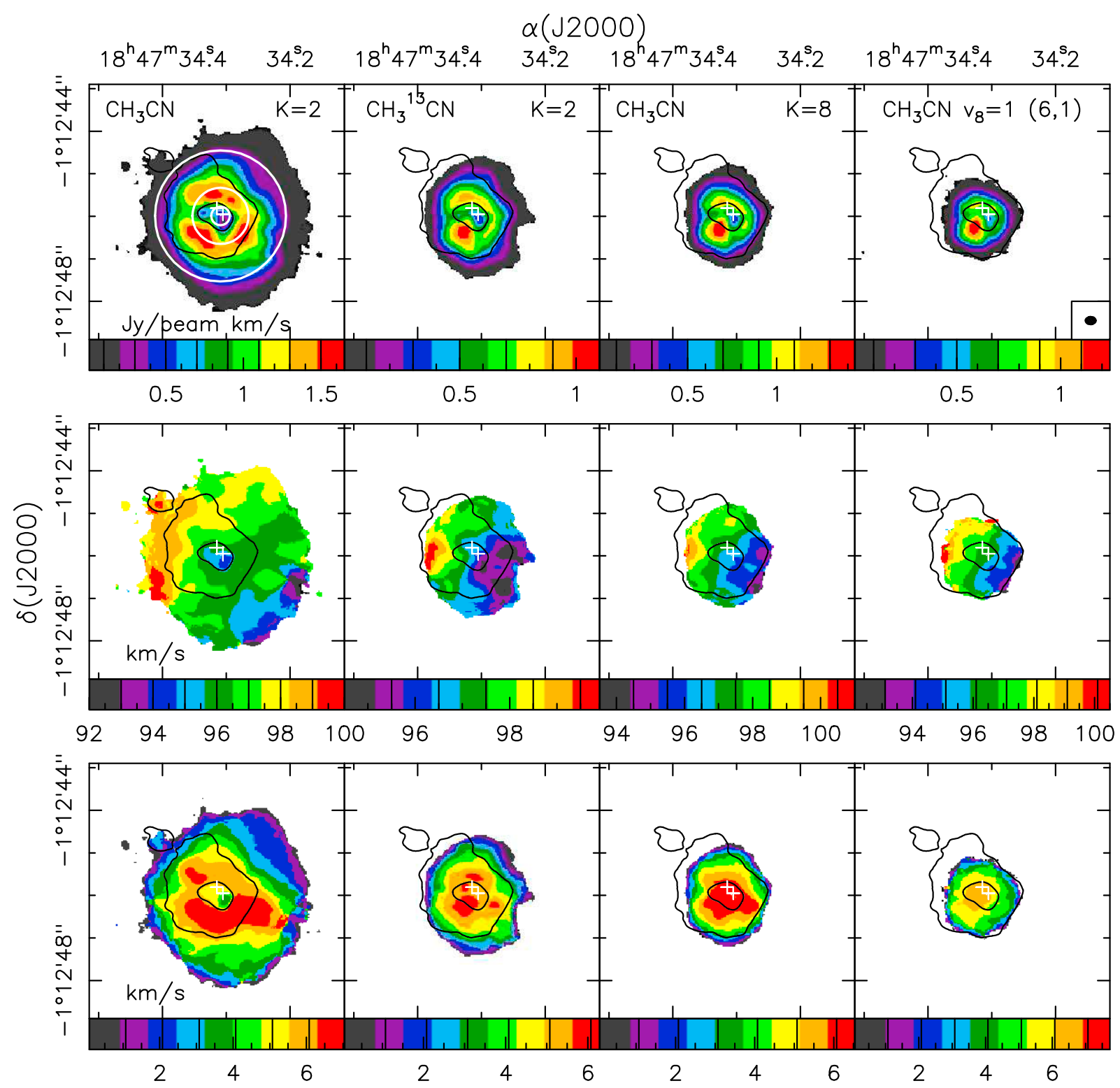

Fig. A.2. Top panels: overlay of the $217 \mathrm{GHz}$ continuum emission (black contours) on the integrated intensity (moment 0) map (colors) of $\mathrm{CH}_{3} \mathrm{CN} K=2, \mathrm{CH}_{3}{ }^{13} \mathrm{CN} K=2, \mathrm{CH}_{3} \mathrm{CN} K=8$, and $\mathrm{CH}_{3} \mathrm{CN} K, l=(6,1) v_{8}=1(12-11)$. The contours correspond to the $5 \sigma$ and $50 \%$ levels. The concentric white circles in the first panel have radii of 0.22 , 0.'66, and 1'.54, (see Sects. 4.1 and 4.2). Middle panels: line velocity (moment 1) maps for the same molecular species. Bottom panels: velocity dispersion (moment 2) maps for the same species. The white crosses indicate the positions of the two compact free-free continuum sources detected by Cesaroni et al. (2010). The synthesized beam is shown in the lower right-hand corner of the top right panel. 
A\&A 615, A141 (2018)
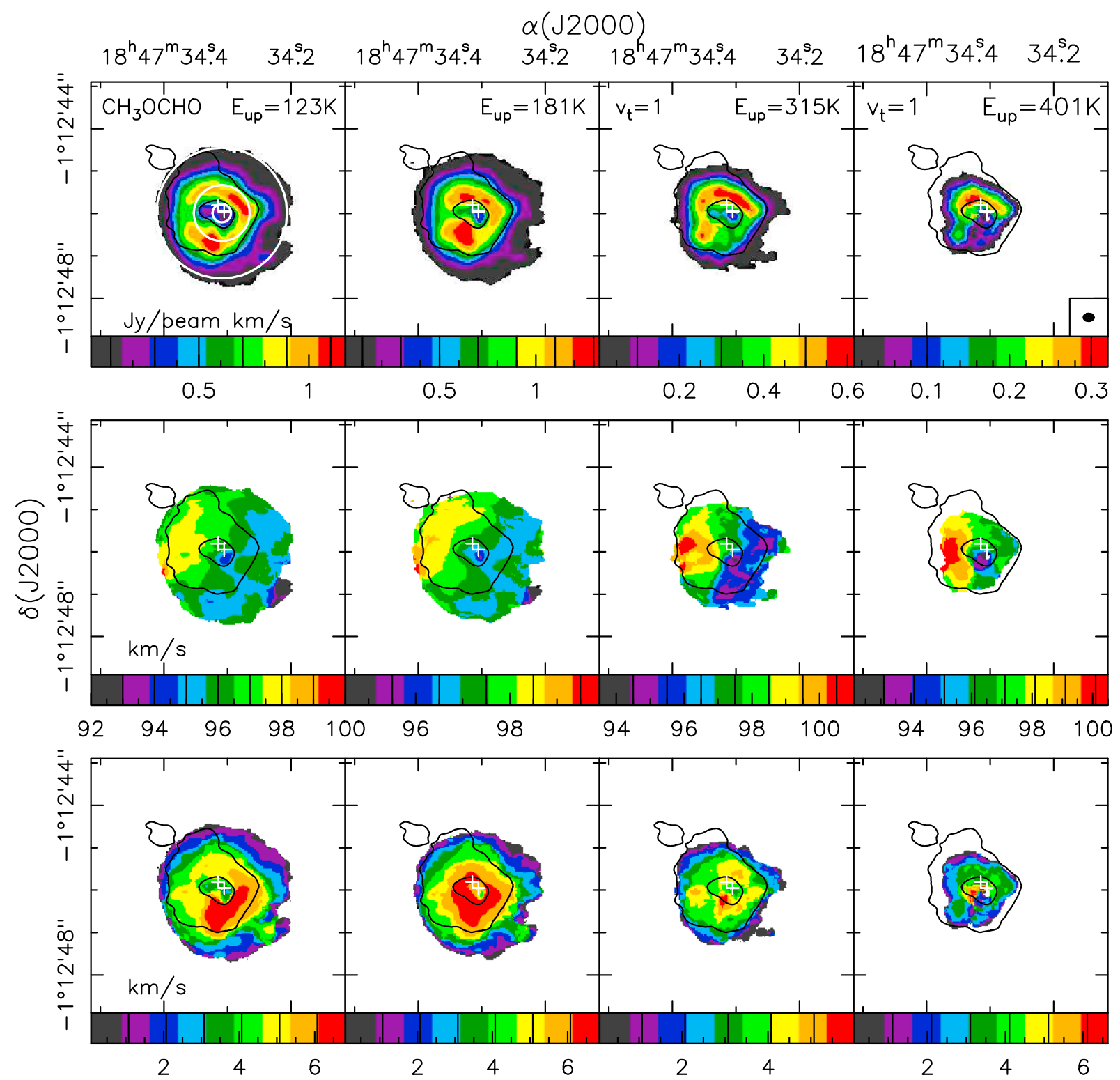

Fig. A.3. Same as Fig. A.2 for $\mathrm{CH}_{3} \mathrm{OCHO}\left(1_{4,16}-18_{4,15}\right) \mathrm{A},\left(18_{11,7}-17_{11,6}\right) \mathrm{A},\left(20_{2,18}-19_{2,17}\right) \mathrm{A} \mathrm{v}_{t}=1$, and $\left(18_{13,6}-17_{13,5}\right) \mathrm{E} \mathrm{v}_{t}=1$. The upper level energies $E_{\text {up }}$ of each transition are indicated in the upper right-hand corner of the top panels. 